\title{
Annotated Translations of Three of the Euler's Papers on Celestial Mechanics
}

\author{
Sylvio R. Bistafa \\ University of São Paulo, São Paulo, Brazil \\ Email: sbistafa@usp.br
}

How to cite this paper: Bistafa, S. R. (2019). Annotated Translations of Three of the Euler's Papers on Celestial Mechanics. Advances in Historical Studies, 8, 252-300. https://doi.org/10.4236/ahs.2019.85018

Received: August 7, 2019

Accepted: December 23, 2019

Published: December 26, 2019

Copyright $\odot 2019$ by author(s) and Scientific Research Publishing Inc. This work is licensed under the Creative Commons Attribution International License (CC BY 4.0).

http://creativecommons.org/licenses/by/4.0/ (c) (i) Open Access

\begin{abstract}
Annotated translations from Latin of three of the Euler's papers on celestial mechanics are presented, which fall into the category of three-body problems. The first translation deals with an exact solution of three bodies that move around the common center of mass and always line up. This is considered the first work from which the three collinear Lagrange points could be obtained. The second translation deals with motions of Sun, Earth and Moon in syzygy and Moon libration as well, where, for the first time, Euler introduces an archaic form of a Fourier sine series expansion to describe the Moon's wagging motion. The last translation relates to a paper that was written with the goal of alleviating astronomical computations of the perturbed motion of the Moon around the Earth by the Sun, ending up with eight coupled differential equations for resolving the perturbed motion of this celestial body. Despite showing great analytical skills, Euler gave no indications on how this system of equations could be solved, which renders his efforts practically useless in the determination of the variations of the nodal line and inclination of the Moon's orbit.
\end{abstract}

\section{Keywords}

Three-Body Problem, Motion of Celestial Bodies, Astronomical Perturbation, Lunar Motion

\section{Introduction}

As earlier as the 1730's and until his death in 1783, Euler wrote more than 60 papers on astronomy, including the motion of planets and comets, astronomical perturbation, eclipses, tides and geophysics. Since many of these works often involve rather lengthy and intricate astronomical computations which, nowadays, are, perhaps, of limited interest, we have chosen to translate works of long 
lasting repercussion such as those related to theoretical and mathematical models to the motion of celestial bodies, and particularly to three-body problems in astronomy. Historically, the first specific three-body problem to receive extended study was the one involving the Moon, the Earth, and the Sun, for which Euler gave a significant contribution.

Although some of Euler's papers on the subject had been written in French, most of them are written in Latin, which may represent a barrier to the modern reader. With the goal of disseminating the works of Euler on the subject, we present here annotated translations from Latin of three of Euler's papers related to three-body problems in astronomy.

"On the rectilinear motion of three bodies mutually attracting each other": in this publication, Euler considers three bodies lying on a straight line, which are attracted to each other by central forces inversely proportional to the square of their separation distance (inverse-square law). Although not explicitly mentioned by Euler, this is an exact solution of three bodies that move around the common center of mass and always line up.

"Considerations on the motion of celestial bodies": in this publication, Euler essentially focuses on the solution of two particular motions of a three-body problem consisting of Sun, Earth and Moon. The first motion, represents a hypothetical situation of these three celestial bodies in perpetual alignment in syzygy - the three-body problem on a straight line. The second motion considered by Euler was Moon libration, when these planets are aligned in regular syzygy.

"An easy method for calculating the motion of celestial bodies perturbed in any manner avoiding astronomical computations": as revealed by its title itself, the goal of this paper is to alleviate the astronomical computations in a typical celestial three-body problem represented by Sun, Earth and Moon. In this work, Euler's approach consists of two parts: geometrical and mechanical. The geometrical part contains most of the analytical developments, in which Euler makes use of Cartesian and spherical trigonometry as well.

\section{On the Rectilinear Motion of Three Bodies Mutually Attracting Each Other (De Motu Rectilineo Trium Corporum se Mutuo Attrahentium, Euler, 1767)}

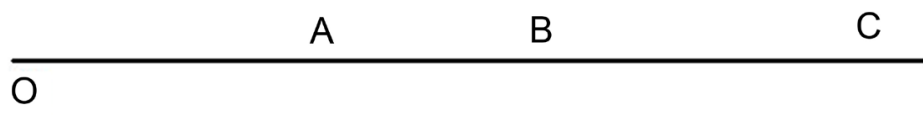

I) Let $A, B, C$ be the masses of three bodies such that their distances to a fixed point $O$ at a given instant of time $t$ is given by

$$
O A=x, O B=y \text { and } O C=z
$$

where, in fact, it is assumed that $y>x$ and $z>y$. Hence, the principles of motion give these three equations:

$$
\text { I) } \frac{\mathrm{dd} x}{\mathrm{~d} t^{2}}=\frac{B}{(y-x)^{2}}+\frac{C}{(z-x)^{2}}
$$




$$
\begin{aligned}
& \text { II) } \frac{\mathrm{dd} y}{\mathrm{~d} t^{2}}=\frac{-A}{(y-x)^{2}}+\frac{C}{(z-y)^{2}} \\
& \text { III) } \frac{\mathrm{dd} z}{\mathrm{~d} t^{2}}=\frac{-A}{(z-x)^{2}}-\frac{B}{(z-y)^{2}}
\end{aligned}
$$

whence two integrable equations are easily derived: the first [integral]

$$
A \mathrm{~d} x+B \mathrm{~d} y+C \mathrm{~d} z=E \mathrm{~d} t
$$

which upon integration results in

$$
A x+B y+C z=E t+F ;^{1}
$$

and the second [integral]

$$
\frac{A \mathrm{~d} x^{2}+B \mathrm{~d} y^{2}+C \mathrm{~d} z^{2}}{\mathrm{~d} t^{2}}=G+\frac{2 A B}{y-x}+\frac{2 A C}{z-x}+\frac{2 B C}{z-y}
$$

whence, because we lack a third integral equation, very little is possible to conclude about the movement.

2) Let us set $x=y-p$ and $z=y+q$, such that $p$ and $q$ are positive quantities; and the first integral [Equation (5)] gives:

$$
(A+B+C) y-A p+C q=E t+F
$$

and thus

$$
\begin{gathered}
y=\frac{A p-C q+E t+F}{A+B+C} ; \mathrm{d} y=\frac{A \mathrm{~d} p-C \mathrm{~d} q+E \mathrm{~d} t}{A+B+C} \\
x=\frac{-(B+C) p-C q+E t+F}{A+B+C} ; \mathrm{d} x=\frac{-(B+C) \mathrm{d} p-C \mathrm{~d} q+E \mathrm{~d} t}{A+B+C} \\
\mathrm{z}=\frac{A p+(A+B) q+E t+F}{A+B+C} ; \mathrm{d} z=\frac{A \mathrm{~d} p+(A+B) \mathrm{d} q+E \mathrm{~d} t}{A+B+C}
\end{gathered}
$$

whence, the second integral [Equation (6)] assumes the following form:

$$
\begin{aligned}
& \frac{A(B+C) \mathrm{d} p^{2}+C(A+B) \mathrm{d} q^{2}+2 A C \mathrm{~d} p \mathrm{~d} q+E E \mathrm{~d} t^{2}}{(A+B+C) \mathrm{d} t^{2}} \\
& =G+\frac{2 A B}{p}+\frac{2 A C}{p+q}+\frac{2 B C}{q}
\end{aligned}
$$

whence, arises one integral equation ${ }^{2}$

$$
\frac{B\left(A \mathrm{~d} p^{2}+C \mathrm{~d} q^{2}\right)+A C(\mathrm{~d} p+\mathrm{d} q)^{2}}{(A+B+C) \mathrm{d} t^{2}}=G+\frac{2 A B}{p}+\frac{2 A C}{p+q}+\frac{2 B C}{q},
$$

${ }^{1}$ From I. $\frac{A \mathrm{~d} x}{\mathrm{~d} t}=\int \frac{A B}{(y-x)^{2}} \mathrm{~d} t+\int \frac{A C}{(z-x)^{2}} \mathrm{~d} t$, which upon multiplication by $\frac{\mathrm{d} x}{\mathrm{~d} t}$ gives $\frac{A \mathrm{~d} x^{2}}{\mathrm{~d} t^{2}}=\int \frac{A B}{(y-x)^{2}} \mathrm{~d} x+\int \frac{A C}{(z-x)^{2}} \mathrm{~d} x$, and then $\frac{A \mathrm{~d} x^{2}}{\mathrm{~d} t^{2}}=\frac{A B}{y-x}+\frac{A C}{z-x}$. And similarly for II and III, yielding: $\frac{B \mathrm{~d} y^{2}}{\mathrm{~d} t^{2}}=\frac{A B}{y-x}+\frac{B C}{z-y}$, and $\frac{C \mathrm{~d} z^{2}}{\mathrm{~d} t^{2}}=\frac{A C}{z-x}+\frac{B C}{z-y}$. Taking the sum over the three equations and introducing the constant of integration $G$ yields $\frac{A \mathrm{~d} x^{2}+B \mathrm{~d} y^{2}+C \mathrm{~d} z^{2}}{\mathrm{~d} t^{2}}=G+\frac{2 A B}{y-x}+\frac{2 A C}{z-x}+\frac{2 B C}{z-y}$. ${ }^{2}$ In the original manuscript, these three last lines have been misplaced at the end of $₫ 3$. 
and observing that the last term $E E$ is included into $G$.

3) Let us make the same proper substitutions into the first second order differential [difference-of-differentials] equations [Equation (1) and Equation (3)], which now result in two [equations]:

$$
\begin{aligned}
& \frac{-(B+C) \mathrm{dd} p-C \mathrm{dd} q}{(A+B+C) \mathrm{d} t^{2}}=\frac{B}{p p}+\frac{C}{(p+q)^{2}} \\
& \frac{A \mathrm{dd} p+(A+B) \mathrm{dd} q}{(A+B+C) \mathrm{d} t^{2}}=\frac{-A}{(p+q)^{2}}-\frac{B}{q q},
\end{aligned}
$$

which [by subtracting Equation (13) from Equation (12)] results in

$$
\frac{\mathrm{dd} p+\mathrm{dd} q}{\mathrm{~d} t^{2}}=\frac{-A-C}{(p+q)^{2}}-\frac{B}{p p}-\frac{B}{q q} .
$$

And then, each element $\mathrm{dd} p$ and $\mathrm{dd} q$ can be expressed separately in the following way

$$
\begin{aligned}
& \text { 1) } \frac{\mathrm{dd} p}{\mathrm{~d} t^{2}}=\frac{-A-B}{p p}-\frac{C}{(p+q)^{2}}+\frac{C}{q q} \\
& \text { 2) } \frac{\mathrm{dd} q}{\mathrm{~d} t^{2}}=\frac{A}{p p}-\frac{A}{(p+q)^{2}}-\frac{B \mp C}{q q}
\end{aligned}
$$

4) Since the solution has been reduced to two differential equations involving $p, q$ and $t$ we should expect that significant advantage is to be obtained, if it were possible to reduce these equations to two others of first order only. This is a unique technique that I have discovered which can be applied in the following manner. I put $q=p u$, and the two differential equations [Equation (15) and Equation (16)] are represented as:

$$
\begin{gathered}
\mathrm{d}\left(\frac{\mathrm{d} p}{\mathrm{~d} t}\right)=\frac{\mathrm{d} t}{p p}\left(-A-B-\frac{C}{(u+1)^{2}}+\frac{C}{u u}\right) \\
\mathrm{d}\left(\frac{u \mathrm{~d} p+p \mathrm{~d} u}{\mathrm{~d} t}\right)=\frac{\mathrm{d} t}{p p}\left(A-\frac{A}{(u+1)^{2}}-\frac{B \mp C}{u u}\right)
\end{gathered}
$$

Now the trick consists of the following substitution $\frac{\mathrm{d} p}{\mathrm{~d} t}=\frac{r}{\sqrt{p}}$ and $\frac{\mathrm{d} q}{\mathrm{~d} t}=\frac{u \mathrm{~d} p+p \mathrm{~d} u}{\mathrm{~d} t}=\frac{s}{\sqrt{p}}$; because it will expose that for these substitutions, the two variables $p$ and $t$ can be eliminated from the calculations, such that only these three [variables] $r, s$ and $u$ are to be determined by their first differentials. Then, in particular, the equation that the integral was found above [Equation (17)] assumes a finite form which reads

$$
\frac{B(A r r+C s s)+A C(r+s)^{2}}{A+B+C}=G p+2 A B+\frac{2 A C}{u+1}+\frac{2 B C}{u},
$$

whose usefulness it will be possible to assess. 
5) Since $\frac{\mathrm{d} p}{\mathrm{~d} t}=\frac{r}{\sqrt{p}}$, then $\mathrm{d} t=\frac{\mathrm{d} p \sqrt{p}}{r}$, whence our second order differential [difference-of-differentials] equations [Equation (18) and Equation (19)] give

$$
\begin{aligned}
& \frac{\mathrm{d} r}{\sqrt{p}}-\frac{r \mathrm{~d} p}{2 p \sqrt{p}}=\frac{\mathrm{d} p}{p r \sqrt{p}}\left(-A-B-\frac{C}{(u+1)^{2}}+\frac{C}{u u}\right) \\
& \frac{\mathrm{d} s}{\sqrt{p}}-\frac{s \mathrm{~d} p}{2 p \sqrt{p}}=\frac{\mathrm{d} p}{p r \sqrt{p}}\left(A-\frac{A}{(u+1)^{2}}-\frac{B \mp C}{u u}\right)
\end{aligned}
$$

or:

$$
\begin{gathered}
\mathrm{d} r=\frac{r \mathrm{~d} p}{2 p}+\frac{\mathrm{d} p}{p r}\left(-A-B-\frac{C}{(u+1)^{2}}+\frac{C}{u u}\right) \\
\mathrm{d} s=\frac{s \mathrm{~d} p}{2 p}+\frac{\mathrm{d} p}{p r}\left(A-\frac{A}{(u+1)^{2}}-\frac{B \mp C}{u u}\right)
\end{gathered}
$$

Moreover, in particular, it will be considered that

$$
u \mathrm{~d} p+p \mathrm{~d} u=\frac{s \mathrm{~d} t}{\sqrt{p}}=\frac{s \mathrm{~d} p}{r},
$$

such that $\frac{\mathrm{d} p}{p}=\frac{r \mathrm{~d} u}{s-r u}$ which when substituted [into Equation (23) and Equation (24)] gives

$$
\begin{aligned}
& \mathrm{d} r(s-r u)=\frac{1}{2} r r \mathrm{~d} u+\mathrm{d} u\left(-A-B-\frac{C}{(u+1)^{2}}+\frac{C}{u u}\right) \\
& \mathrm{d} s(s-r u)=\frac{1}{2} r s \mathrm{~d} u+\mathrm{d} u\left(A-\frac{A}{(u+1)^{2}}-\frac{B \mp C}{u u}\right)
\end{aligned}
$$

which when combined give:

$$
\frac{1}{2} r(r \mathrm{~d} s-s \mathrm{~d} r)+\mathrm{d} s\left(-A-B-\frac{C}{(u+1)^{2}}+\frac{C}{u u}\right)-\mathrm{d} r\left(A-\frac{A}{(u+1)^{2}}-\frac{B \mp C}{u u}\right)=0
$$

6) We see that we have two first-order differential equations [Equation (26) and Equation (27)] among three variables $r, s$ and $u$, whence if it were possible to determine $r$ and $s$ in terms of $u$, and then one would have the complete solution of the problem. Thence, in fact, $p$ would become known from the formula $\frac{\mathrm{d} p}{p}=\frac{r \mathrm{~d} u}{s-r u}$, and hence furthermore $q=p u$. Thereafter, the particular time $t$ would be given from the equation $\mathrm{d} t=\frac{\mathrm{d} p \sqrt{p}}{r}=\frac{p \mathrm{~d} u}{s-r u}$; and finally, for a given time $t$, the distances $x, y, z$ would be obtained as given in $₫ 2$.

7) Since the two differential equations [Equation (26) and Equation (27)] found are 


$$
\begin{gathered}
\mathrm{d} r(s-r u)=\frac{1}{2} r r \mathrm{~d} u+\mathrm{d} u\left(-A-B-\frac{C}{(u+1)^{2}}+\frac{C}{u u}\right) \\
\mathrm{d} s(s-r u)=\frac{1}{2} r s \mathrm{~d} u+\mathrm{d} u\left(A-\frac{A}{(u+1)^{2}}-\frac{B-C}{u u}\right)
\end{gathered}
$$

then, it is clear that both are satisfied by taking the quantity $u$ as constant and $s-r u=0$, whence a particular solution is obtained. If $u=\propto$ and $s=\propto r$ [then from Equation (28) we have that:]

$$
-(A+B) \propto-\frac{C \propto}{(\propto+1)^{2}}+\frac{C}{\propto}=A-\frac{A}{(\propto+1)^{2}}-\frac{B \mp C}{\propto \propto},
$$

or

$$
0=A\left(\propto+1-\frac{1}{(\propto+1)^{2}}\right)+B\left(\propto-\frac{1}{\propto \propto}\right)+C\left(\frac{\propto}{(\propto+1)^{2}}-\frac{1}{\propto}-\frac{1}{\propto \propto}\right),
$$

or else

$$
0=A \frac{\left[(\propto+1)^{3}-1\right]}{(\propto+1)^{2}}+\frac{B\left(\propto^{3}-1\right)}{\propto \propto}+\frac{C\left[\propto^{3}-(\propto+1)^{3}\right]}{\propto \propto(\propto+1)^{2}}
$$

hence,

$$
C(1+3 \propto+3 \propto \propto)=A \propto^{3}(\propto \propto+3 \propto+3)+B(\propto+1)^{2}\left(\propto^{3}-1\right) .
$$

Thus, it is possible to determine the quantity $\propto$ from this equation of the fifth degree:

$$
\begin{aligned}
& (A+B) \propto^{5}+(3 A+2 B) \propto^{4}+(3 A+B) \propto^{3}-(B+3 C) \propto^{2}{ }_{3} \\
& -(2 B+3 C) \propto-B-C=0
\end{aligned}
$$

Thence, truly from the relation between $r$ and $p$ [Equation (21)] this equation is obtained

$$
\mathrm{d} r=\frac{r \mathrm{~d} p}{2 p}+\frac{\mathrm{d} p}{p r}\left(-A-B-\frac{C}{(\propto+1)^{2}}+\frac{C}{\propto \propto}\right)
$$

or by putting $A+B+\frac{C}{(\propto+1)^{2}}-\frac{C}{\propto \propto}=\frac{1}{2} D$, [then, from Equation (34) we have]

$$
2 \mathrm{~d} r=\frac{\mathrm{d} p}{p}\left(r-\frac{D}{r}\right) \text { or } \frac{\mathrm{d} p}{p}=\frac{2 r \mathrm{~d} r}{r r-D},
$$

which [upon integration of Equation (35b)] gives

$$
p=\beta(r r-D),
$$

[where $\zeta$ is a constant of integration], and then [since $q=p u=p \propto$ ]

$$
q=\propto \beta(r r-D),
$$

and [since] $\mathrm{d} t=\frac{\mathrm{d} p \sqrt{p}}{r}$ or $\mathrm{d} t=2 \beta \mathrm{d} r \sqrt{\beta(r r-D)}$, hence 


$$
t=\beta r \sqrt{\beta(r r-D)}-\beta^{2} D \int \frac{\mathrm{d} r}{\sqrt{\beta(r r-D)}}
$$

8) This particular case, in which the solution succeeds, deserves to be unfolded carefully. Firstly, I observe that the value of $\propto$, since it is obtained from an equation of the fifth degree, is unique and always a positive real quantity, because there is only one sign variation ${ }^{3}$, and then, of course, there is no reason for any ambiguity; however, the value of this $\propto$ can be seen to depend on the masses of the three bodies $A, B, C$. Once the number $\propto$ having been found, we get the quantity $D=2(A+B)-\frac{2 C(2 \propto+1)}{\propto \propto(\propto+1)^{2}}$, where it should be observed that the quantity $D$ can never vanish. In fact, if $D=0$, then $B=\frac{C(2 \propto+1)}{\propto \propto(\propto+1)^{2}}-A$, which, when substituted [into Equation (32)] would give:

$$
\begin{aligned}
& C(1+3 \propto+3 \propto \propto) \\
& =A \propto^{3}(\propto \propto+3 \propto+3)+\frac{C(2 \propto+1)\left(\propto^{3}-1\right)}{\propto \propto}-A(\propto+1)^{2}\left(\propto^{3}-1\right) \\
& \frac{C}{\propto \propto}\left(\propto^{4}+2 \propto^{3}+\propto \propto+2 \propto+1\right)=A\left(\propto^{4}+2 \propto^{3}+\propto \propto+2 \propto+1\right)
\end{aligned}
$$

and, therefore, $C=A \propto \propto$ and $B=\frac{A(2 \propto+1)}{(\propto+1)^{2}}-A=\frac{-A \propto \propto}{(\propto+1)^{2}}$, and then, $B$ would be a negative mass, which is absurd. Even less possible is that the quantity $D$ could be negative. In fact, assuming [that $\frac{D}{2}=-\Delta$, where $\Delta$ is a positive quantity]:

$$
B=\frac{C(2 \propto+1)}{\propto \propto(\propto+1)^{2}}-A-\Delta,
$$

it would then give [when substituted into Equation (32)]:

$$
\frac{C}{\propto \propto}=A-\frac{\Delta(\propto+1)^{2}\left(\propto^{3}-1\right)}{\propto^{4}+2 \propto^{3}+\propto \propto+2 \propto+1},
$$

hence [by isolating $A$ in the first term of Equation (42), and substituting the resulting expression into Equation (41)]

$$
B=\frac{C(2 \propto+1)}{\propto \propto(\propto+1)^{2}}-\frac{C}{\propto \propto}-\frac{\Delta\left(\propto^{5}+3 \propto^{4}+3 \propto^{3}\right)}{\propto^{4}+2 \propto^{3}+\propto \propto+2 \propto+1},
$$

and then, $B$ would be a much more negative quantity, since it is necessary that the value of $\propto$ itself be positive.

${ }^{3}$ Descartes' Rule of Signs states that if the terms of a single-variable polynomial with real coefficients are ordered by descending variable exponent, then the number of positive roots of the polynomial is either equal to the number of sign differences between consecutive nonzero coefficients, or is less than it by an even number. 
9) Then, since it is necessary that the quantity $D$ be positive, it can be assumed that $D=a a$, and if also the number $\propto$ is considered as given, therefore the masses of the three bodies will be obtained as [from Equation (43), with $\Delta=\frac{a a}{2}$ ]:

$$
B=\frac{\left(\propto^{5}+3 \propto^{4}+3 \propto^{3}\right) a a}{2\left(\propto^{4}+2 \propto^{3}+\propto \propto+2 \propto+1\right)}-\frac{C}{(\propto+1)^{2}} ;
$$

and [from Equation (42), with $\Delta=-\frac{a a}{2}$ ]:

$$
A=\frac{C}{\propto \propto}-\frac{(\propto+1)^{2}\left(\propto^{3}-1\right) a a}{2\left(\propto^{4}+2 \propto^{3}+\propto \propto+2 \propto+1\right)},
$$

from which it is necessary that the quantity $\frac{2 C\left(\propto^{4}+2 \propto^{3}+\propto \propto+2 \propto+1\right)}{\propto \propto(\propto+1)^{2} a a}$ be bound between the limits $(\propto+1)^{3}-1$ and $\propto^{3}-1$. Then, once the quantity aa with the number $\propto$ are introduced into the calculations, two cases should be examined, according to the sign of the quantity $\beta$ (positive or negative), which we shall examine separately.

Case I.

10) Be first $\beta=n n$, and then $p=n n(r r-a a)$ [from Equation (36)] and $q=\propto n n(r r-a a)$ [from Equation (37)], and by putting the constants $E$ and $F$ equal to zero, the locations of the three bodies $A, B, C$, which center of gravity is now located at $O$, are defined by $r$ such that:

$$
\begin{gathered}
x=O A=\frac{-n n(r r-a a)}{A+B+C}(B+C+C \propto), \\
y=O B=\frac{n n(r r-a a)}{A+B+C}(A-C \propto), \\
z=O C=\frac{n n(r r-a a)}{A+B+C}(A+(A+B) \propto) .
\end{gathered}
$$

Yet, the relation between $r$ and the time $t$ is [Equation (38)]

$$
t=n^{3} r \sqrt{r r-a a}-n^{3} a a \int \frac{\mathrm{d} r}{\sqrt{r r-a a}},
$$

or

$$
t=n^{3} r \sqrt{r r-a a}-n^{3} a a \ln \left|\frac{r+\sqrt{r r-a a}}{\Delta}\right| .
$$

Assuming that the constant $\Delta=a$, then, for the time $t=0, r=a$, meaning that all bodies are concentrated in the center of gravity $[O]$, whence they will be driven out with an almost infinite velocities, and then, these [distances] are similar to each other as the quantities of: $-B-C-C \propto, A-C \propto, A+(A+B) \propto$; also, with the passage of time $t$ the quantity $r$ increases even more; however, for any other time, the velocity of each of the bodies becomes known from the formula 
$\frac{\mathrm{d} t}{\mathrm{~d} r}=2 n^{3} \sqrt{r r-a a}$. However, noting that the inter body distances preserve the same proportion.

Case II.

11) Be now $\beta=-n n$, and then $p=n n(a a-r r)$ [from Equation (36)] and $q=\propto n n(a a-r r)$ [from Equation (37)], and the locations of the bodies are defined by $r$ from:

$$
\begin{gathered}
x=O A=\frac{-n n(a a-r r)}{A+B+C}(B+(1+\propto) C), \\
y=O B=\frac{n n(a a-r r)}{A+B+C}(A-C \propto), \\
z=O C=\frac{n n(a a-r r)}{A+B+C}(A(\propto+1)+B \propto) .
\end{gathered}
$$

On the other hand, for the time $t$ to be obtained, $t=2 n^{3} \mathrm{~d} r \sqrt{(a a-r r)}$, or

$$
t=n^{3} r \sqrt{a a-r r}+n^{3} a a \int \frac{\mathrm{d} r}{\sqrt{(a a-r r)}}
$$

hence,

$$
t=n^{3} r \sqrt{a a-r r}+n^{3} a a \sin ^{-1}\left(\frac{r}{a}\right) .
$$

But, by putting $\sin ^{-1}\left(\frac{r}{a}\right)=\phi$, such that $r=a \sin \phi$, then

$t=n^{3} a a(\phi+\sin \phi \cos \phi)$, and at any time, the inter distances are proportional to $\cos ^{2} \phi{ }^{4}$ Whence, if at the beginning when $t=0$, also $\phi=0$, thus $r=0$, and $\frac{\mathrm{d} t}{\mathrm{~d} r}=2 n^{3} a$, then the distances will be:

$$
\begin{gathered}
x=O A=\frac{-n n a a}{A+B+C}(B+(1+\propto) C), \\
y=O B=\frac{n n a a}{A+B+C}(A-C \propto), \\
z=O C=\frac{n n a a}{A+B+C}(A(\propto+1)+B \propto),
\end{gathered}
$$

and in that place the bodies are at rest. On the other hand, once it has been assumed that $\phi=90^{\circ}$, or after the time $t=n^{3} a a \cdot 90^{\circ}$ has elapsed, the bodies approach the center of gravity with infinity velocity. ${ }^{5}$

\footnotetext{
${ }^{4}$ According to Equations (51)-(53), the distances are proportional to $\left(a^{2}-r^{2}\right)=\left(a^{2}-a^{2} \frac{r^{2}}{a^{2}}\right)=a^{2}\left(1-\sin ^{2} \phi\right)=a^{2} \cos ^{2} \phi$.

${ }^{5}$ Since $\mathrm{d} t=-2 n n \mathrm{~d} r \sqrt{n n(a a-r r)}$, or $\frac{\mathrm{d} r}{\mathrm{~d} t}=-\frac{1}{2 n^{3} \sqrt{(a a-r r)}}$, which for $\phi=90^{\circ}(r=a)$ gives $\frac{\mathrm{d} r}{\mathrm{~d} t}=-\infty$
} 


\section{Applications by the Translator}

For $A=B=C=1$ unit, the fifth order polynomial [Equation (33)] reduces to

$$
2 \propto^{5}+5 \propto^{4}+4 \propto^{3}-4 \propto^{2}-5 \propto-2=0
$$

which, by inspection, gives $\propto=1$ as the single positive root.

For Case I, and from Equations (46)-(48), the positions of these masses are given by $x=-n n(r r-a a), y=0$, and $z=n n(r r-a a)$, which show that one of the masses occupies the center of gravity, whereas the two other masses occupy symmetrical positions in relation to the center of gravity, and that these positions depend on $(r r-a a)$.

For Case II, and when $t=0$, the motion begins in a symmetrical configuration of the masses in relation to the center of gravity, however, according to Equations (56)-(58), the positions of these masses are now given by $x=-n n a a$, $y=0$, and $z=n n a a$. For any other instant of time, the positions of the masses will be given by $x=-n n(a a-r r), y=0$, and $z=n n(a a-r r)$, which show that the symmetrical configuration is preserved, however, the positions of the masses now depend on $(a a-r r)$ instead.

The conclusion is that for these particular cases, the configuration of the masses during the motion is such that one of the masses occupies the center of gravity, with the two other masses remaining on the same straight line, and moving symmetrically around the center of gravity of the system. This is considered the first work from which the three collinear Lagrange points could be obtained, where the parameter that controls the distances among the bodies was found to be given by a quintic function.

A practical application of these results is to find, for instance, the Earth-Moon three collinear Lagrange points for satellite location. The figure below provides a graphical interpretation of the Lagrange points, for the application of the quintic equation [Equation (33)]. Considering that the Earth-Moon distance $d_{\text {Earth-Moon }}=384400 \mathrm{~km}$, and that the mass of the Earth equals $5.98 \times 10^{24} \mathrm{~kg}$, the mass of the Moon equals $7.34 \times 10^{22} \mathrm{~kg}$, and the mass of the satellite equals zero, approximately, then the solution of the quintic equation gives: $\propto_{1}=0.17737$, $\propto_{2}=0.167417, \propto_{3}=1.00711$, for the Lagrange points $L_{1}, L_{2}, L_{3}$, respectively. These values gives the following distances from the Earth as: $L_{1}=326490 \mathrm{~km}$, $L_{2}=448755 \mathrm{~km}, L_{3}=381686 \mathrm{~km}$. These values can also be obtained by the following approximate expressions that have been used:

$$
\begin{gathered}
L_{1}=\frac{d_{\text {Earth-Moon }}}{1+r_{1}}, \text { with } r_{1} \approx \sqrt[3]{\frac{m_{\text {Moon }}}{3 m_{\text {Earth }}}}=0.159617 \Rightarrow L_{1}=331488 \mathrm{~km} \\
L_{2}=d_{\text {Earth-Moon }}\left(1+r_{2}\right), \text { with } r_{2} \approx \sqrt[3]{\frac{m_{\text {Moon }}}{3 m_{\text {Earth }}}}=0.159617 \Rightarrow L_{2}=445757 \mathrm{~km} \\
L_{3}=\frac{d_{\text {Earth-Moon }}}{r_{3}}, \text { with } r_{3} \approx 1+\frac{5 m_{\text {Moon }}}{12 m_{\text {Earth }}}=1.005083 \Rightarrow L_{3}=382456 \mathrm{~km}
\end{gathered}
$$



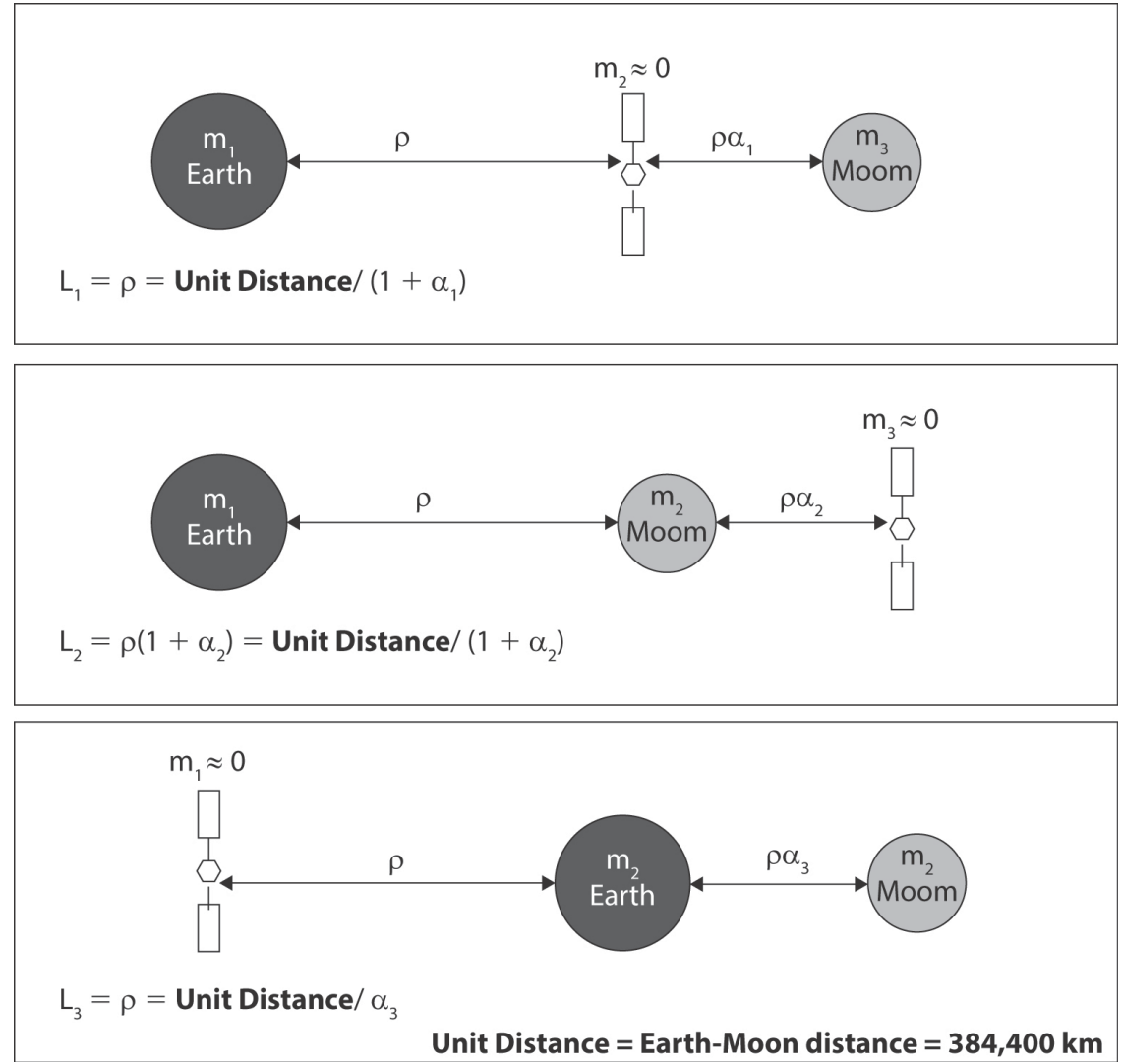

Graphical interpretation of the Lagrange points, for the application of the quintic equation (Adapted from: Problem Set 1 Solution, Satellite Engineering, David W. Miller and John E. Keesee. Available at https://ocw.mit.edu/courses/aeronautics-and-astronautics/16-851-satellite-engin eering-fall-2003/assignments/ps1 nn solution.pdf, Accessed on Aug. 30, 2019)

\section{Considerations on the Motion of Celestial Bodies (Considerationes de Motu Corporum Coelestium, Euler, 1766)}

I) Although there is no doubt that the laws of motion of celestial bodies observed by Kepler and confirmed by Newton have brought very great gains to [the discipline of] Astronomy, nevertheless it is certain that no body in the heavens is met with that in its own motion follows these laws perfectly, since, instead, in all [of the motions of these bodies] deviations from these laws, that are by no means slight, are detected. Of course, it is true that the cause of all heavenly motions resides in the mutual attraction of these bodies, by which each and every [body] is attracted toward each of the others singly by forces consisting of a ratio composed directly by the un-squared [amount] of the masses [of the bodies], and inversely by the squared [amounts] of the distances. However, it is always convenient to consider that one force stands out among the remaining, and thus, the motion would approximately follows Kepler's rules; and then the relatively very small effect arising from the others can be determined by methods of approxi- 
mation. Without this simplification, we would be at the utmost ignorance about the celestial motion, since to date no method has been discovered by the application of which the motion, of three or more bodies mutually attracting each other, might require to be ascribed; unless, perchance, one force surpasses the others.

2) Yet indeed this case-in which [case] alone Geometers do not squander their work at all points in vain-cannot be taken as conclusive since the method of approximation itself, which Geometers are accustomed to use, is bound up with a great many difficulties besides, and an unlimited multitude of small perturbations is neglected, by which [fact] it becomes so that this approximation by itself [only] minimally carries through the business [of determining the motions], but on the contrary, for it to be completed, still more supports are desired. Wherefore, although from this Theory the motion of the Moon is determined accurately enough, that [fact of sufficient accuracy] ought to be ascribed more to special circumstances that obtain for the Moon than to any perfection to which [perfection] a general Theory would be required to measure up. For if the Moon were two or three times as far from the Earth, or if its orbit were more eccentric, then all the labors endured to this point would be lacking in all fruit [because they would be inapplicable], and by the way, not even its motion could be recalled to any fixed rule.

3) Therefore, much has stood before the Theory of Astronomy to be considered, for instance, if under the fictitious hypothesis that in case the Moon were much away from the earth, it would be certainly an excess to think that its motion could be evaluated with the maximum aid of this science. If, for instance, the Moon would have been a hundred times more distant from the earth, there is no doubt that the laws of motion of the main planet would no longer be followed as if it were a satellite of the earth, as one would expect. But if, on the other hand, the distance were ten times greater, its motion could then be compared, so that no doubt would remain, even with primary or secondary planets being added. To such an extent that it certainly would disagree from all the motions observed in the sky, such that it can hardly give an idea even on how the average motion can be resolved. Perhaps, innumerable observations could reveal a certain law, from which, in a subsequent application, it can somehow give a clear prediction; however, by no means so evident, to such an extent that the Theory that should explain this type of motion may not be adapted. The very wise creator is seen to have being mindful of our weakness, because none of the bodies placed in the sky are such that their motion could be described neither by the law of the main planets nor of the satellites.

4) This sort of research, which is seen not to surpass the strength of the human mind, is certainly not suited to be undertaken hastily, but on the contrary, it will require that our efforts be undertaken step by step. Then, the general problem of three bodies mutually attracted to each other will be conveniently restricted to the case where one of the masses almost vanishes in front of the two remaining, where it is agreed that certainly it will be convenient that the two 
larger bodies are set in motion according to Kepler's laws, and that every perturbation on the third [body] is disconsidered, and in case its position and motion will be compared since the beginning, such that if it is attracted to both larger [bodies] with almost equal force, we shall have in this manner a case, whose investigation demands a distinctly new approach. A great deal is lacking to venture an approach towards this problem before fatiguing in vain to unfold it, as I am forced to admit; however, in fact, this is a complete singular case as I have already observed, and with a remarkable simplification, in which the motion of the Moon would appear constantly connected or in opposition to the Sun, which is the case to be considered, with great utility in this very difficult matter should not be abandoned, and by no means to be seen with indifference.

5) Hence, the motion of the Sun and the Moon seen from Earth is assumed to take place in the ecliptic plane $e^{6}$, with the earth resting in $T$, and after a certain time has elapsed, I place the Sun in $S$, the Moon in $L$, and after laying a fixed straight line $T A$, directed to the First Star of Aries $^{7}$, I ascribe the following angles: $A T S=\theta, \quad A T L=\phi$, and $S T L=\phi-\theta=\eta$, and the distances $T S=u, T L=v$ and $L S=\sqrt{u u-2 u v \cos \eta+v v}=z$. Be further the mean longitude of the $\operatorname{Sun}^{8}=$ $\zeta$, and its mean distance from the Earth $=a$, and from these we have for the motion of the Sun in its canonical form?

$$
\frac{2 \mathrm{~d} u \mathrm{~d} \theta+u \mathrm{dd} \theta}{\mathrm{d} \zeta^{2}}=0 \text { and } \frac{\mathrm{dd} u-u \mathrm{~d} \theta^{2}}{\mathrm{~d} \zeta^{2}}+\frac{a^{3}}{u^{2}}=0,
$$

and for the motion of the moon ${ }^{10}$ :

$$
\begin{gathered}
\frac{2 \mathrm{~d} v \mathrm{~d} \phi+v \mathrm{dd} \phi}{\mathrm{d} \zeta^{2}}-\frac{a^{3}}{u^{2}}\left(1-\frac{u^{3}}{z^{3}}\right) \sin \eta=0, \text { and } \\
\frac{\mathrm{dd} v-v \mathrm{~d} \phi^{2}}{\mathrm{~d} \zeta^{2}}+\frac{n^{2} c^{3}}{v^{2}}+\frac{a^{3} v}{z^{3}}+\frac{a^{3}}{u^{2}}\left(1-\frac{u^{3}}{z^{3}}\right) \cos \eta=0
\end{gathered}
$$

where $c$ is the mean distance which the Moon is solicited by the force of the Earth, and for the mean motion of revolution, there is a $n: 1$ relation between the mean motion of the Moon and the mean motion of the Sun. Besides, regarding the differentials of the second degree, it should be noticed that the element $\mathrm{d} \zeta$ is assumed to be constant.

\footnotetext{
${ }^{6}$ The ecliptic plane contains most of the objects which are orbiting the sun, and is tilted with respect to the Earth's spin axis at $23.5^{\circ}$.

${ }^{7}$ The First Star of Aries (or First Point of Aries), also known as the Cusp of Aries, is the location of the vernal equinox.

${ }^{8}$ The Sun's ecliptical longitude is defined as the angle subtended at the earth between the vernal equinox and the Sun. The mean longitude is the ecliptical longitude that the planet would have if the orbit were a perfect circle.

${ }^{9}$ The development of these equations can be found in E112-Recherches sur le mouvement des corps célestes en general.

${ }^{10}$ The development of these equations can be found in L. Euler, Considerationes de theoria motus lunae perficienda et imprimis de eius variatione, Novi Commentarii Academie Scientiarum Imperialis Petropolitanae, Tom. XIII, pro Anno 1768.
} 


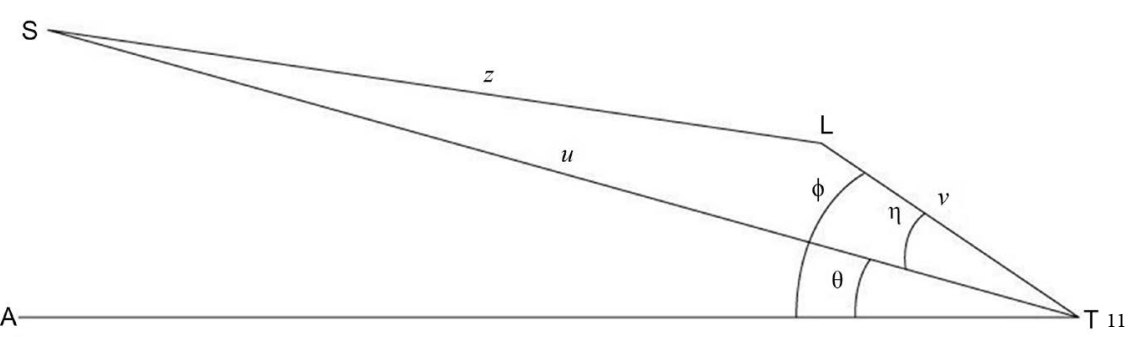

6) All the difficulties in the resolution of both equations, consist on finding at any instant of time, the mean longitude of the Sun $\zeta$, as well as the distance $v$, and the angle $\phi$. Since so far, in general, the Geometers cannot proceed in their work, unless in case in which the distance $v$ is much smaller than $u$ and the number $n$ is rather big, suitable approximations have been found, yet much is still justly needed in this matter. However this pair of equations in a general aspect, without any consideration to the Moon dwelling, which certainly has to be explained, can solve the problem completely. Such motion can take place in the sky, and it is in our ability to know it completely, even if its reasoning does not agree at all with the regular motion.

7) First of all, I should observe that these two equations admit a closed form solution for the case in which $\eta=0$, or $\phi=\theta$, when the Moon is seen to be in continuous communication with the Sun. Since $\sin \eta=0$, and $\cos \eta=1$, then $z=u-v$, and our equations will assume the following forms:

$$
\begin{gathered}
\frac{2 \mathrm{~d} v \mathrm{~d} \theta+v \mathrm{dd} \theta}{\mathrm{d} \zeta^{2}}=0, \text { and } \\
\frac{\mathrm{dd} v-v \mathrm{~d} \theta^{2}}{\mathrm{~d} \zeta^{2}}+\frac{n^{2} c^{3}}{v^{2}}+\frac{a^{3} v}{(u-v)^{3}}+\frac{a^{3}}{u^{2}} \cdot \frac{-3 u^{2} v+3 u v^{2}-v^{3}}{(u-v)^{3}}=0 \\
\text { or } \frac{\mathrm{dd} v-v \mathrm{~d} \theta^{2}}{\mathrm{~d} \zeta^{2}}+\frac{n^{2} c^{3}}{v^{2}}+\frac{a^{3} v\left(2 u^{2}-3 u v+v^{2}\right)}{u^{2}(u-v)^{3}}=0,
\end{gathered}
$$

which can be immediately compared with the given formulas for the motion of the Sun considering that $v=\alpha u$, which it is certainly satisfied by the prior established equations. Hence the other equation for the Moon will be transformed into

$$
\frac{\alpha\left(\mathrm{dd} u-u \mathrm{~d} \theta^{2}\right)}{\mathrm{d} \zeta^{2}}+\frac{n^{2} c^{3}}{\alpha^{2} u^{2}}+\frac{\alpha a^{3}\left(2-3 \alpha+\alpha^{2}\right)}{(1-\alpha)^{3} u^{2}}=0 .
$$

And since the other equation for the Sun is

$$
\frac{\mathrm{dd} u-u \mathrm{~d} \theta^{2}}{\mathrm{~d} \zeta^{2}}+\frac{a^{3}}{u^{2}}=0
$$

it is necessary that

$$
\alpha a^{3}=\frac{n^{2} c^{3}}{\alpha^{2}}-\frac{\alpha a^{3}\left(2-3 \alpha+\alpha^{2}\right)}{(1-\alpha)^{3}}
$$

\footnotetext{
${ }^{11}$ Angles were added to this figure by the Translator to facilitate the comprehension.
} 
or

$$
\frac{n^{2} c^{3}}{\alpha^{2} a^{3}}-\frac{3 \alpha-3 \alpha^{2}+\alpha^{3}}{(1-\alpha)^{2}}
$$

where, since $\frac{n^{2} c^{3}}{a^{3}}$ is a constant quantity, let us put for conciseness $\frac{n^{2} c^{3}}{a^{3}}=m$, and then

$$
m(1-\alpha)^{2}=\alpha^{2}\left(3 \alpha-3 \alpha^{2}+\alpha^{3}\right)
$$

or

$$
m(1-\alpha)^{2}=\alpha^{2}-\alpha^{2}(1-\alpha)^{3} .
$$

Once putted $2-\propto=x$, then $m x^{2}=\left(1-x^{3}\right)(1-x)^{2}$, or

$$
1-2 x+x^{2}-m x^{2}-x^{3}+2 x^{4}-x^{5}=0 \cdot .^{12}
$$

8) Then, it will be necessary the determination of the number $\alpha$ or $x$ from an equation of the fifth degree, which for its resolution, it is necessary to first observe that $m$ should be a rather small fraction, and then

$$
m(1-\alpha)^{2}=3 \alpha^{3}-3 \alpha^{4}+\alpha^{5},
$$

likewise, it is evident that $\alpha$ will also have a small value, which can be approximated by $\propto=\sqrt[3]{\frac{m}{3}}=\frac{c}{a} \sqrt[3]{\frac{n^{2}}{3}}$, or more precisely $\propto=\sqrt[3]{\frac{m}{3}}-\frac{1}{3} \sqrt[3]{\frac{m^{2}}{9}}-\frac{1}{27} m+\frac{1}{81} m \sqrt[3]{\frac{m}{3}}$. However, a first approximation gives $v=\frac{c u}{a} \sqrt[3]{\frac{n^{2}}{3}}$, whence, since $u=a$, and $n^{2}=175,{ }^{13}$ gives, $v=4 c$, approximately; or if the Moon were four times more distant from us, a motion of this kind would have been possible to exist, such that it [the Moon] would appear always connected to the Sun. It would then be possible to regard a Satellite of the Earth as if it were the Moon, and its motion would be most regular, however, deviating from the rules of Kepler the more close to the Sun than to the Earth, though it may revolved with the same time, because the force of the Earth in relation to the force of the Sun is reduced in the same proportion, although it may linger with a longer periodic time. Because the distance to the earth would be almost four times greater than the distance that Moon actually stands apart, as much as a limit would permit, so that bodies far more removed from the principal planets, in fact closer to the satellites of the Earth should permit. Similar limits in relation to other planets will be possible to be established.

\footnotetext{
${ }^{12} \mathrm{~A}$ fifth degree polynomial was also obtained by Euler in E327-De motu rectilineo trium corporum se mutuo attrahentium (see Section 2). In this publication, Euler considers three bodies lying on a straight line, which are attracted to each other by central forces inversely proportional to the square of their separation distance (inverse-square law).

${ }^{13}$ The Moon completes an orbit around the Earth once every 27.32 days. The Earth takes a year (365 days) to revolve around the Sun. Therefore, in a year period, the Moon completes $n=365 / 27.32=13.36$ revolutions around the Earth, and then $n^{2}=178.5$.
} 
9) As the evolved case of a permanent continuous communication with the Sun, indeed in continuous opposition, gives a similar case. In this case, let us put $\eta=180^{\circ}$, and then $\sin \eta=0, \cos \eta=-1$, and $\phi=180^{\circ}+\theta$, and then $\mathrm{d} \phi=\mathrm{d} \theta$, and also $z=u+v$. Thus, the equations for the motion of the Moon will assume the following forms:

$$
\begin{gathered}
\frac{2 \mathrm{~d} v \mathrm{~d} \theta+v \mathrm{dd} \theta}{\mathrm{d} \zeta^{2}}=0, \text { and } \\
\frac{\mathrm{dd} v-v \mathrm{~d} \theta^{2}}{\mathrm{~d} \zeta^{2}}+\frac{n^{2} c^{3}}{v^{2}}+\frac{a^{3} v}{(u+v)^{3}}-\frac{a^{3}}{u^{2}}\left(1-\frac{u^{3}}{(u+v)^{3}}\right)=0,
\end{gathered}
$$

and the latter one reducing to the following:

$$
\frac{\mathrm{dd} v-v \mathrm{~d} \theta^{2}}{\mathrm{~d} \zeta^{2}}+\frac{n^{2} c^{3}}{v^{2}}-\frac{a^{3}}{u^{2}}-\frac{a^{3}}{(u+v)^{2}}=0
$$

First, considering also the motion of the Sun, gives at once $v=\alpha u$, which transform this equation into

$$
\frac{\propto\left(\mathrm{dd} u-u \mathrm{~d} \theta^{2}\right)}{\mathrm{d} \zeta^{2}}+\frac{n^{2} c^{3}}{\propto^{2} u^{2}}-\frac{a^{3}}{u^{2}}-\frac{a^{3}}{(1+\propto)^{2} u^{2}}=0 .
$$

Yet, for the motion $\frac{\mathrm{dd} u-u \mathrm{~d} \theta^{2}}{\mathrm{~d} \zeta^{2}}=-\frac{a^{3}}{u^{2}}$, which transforms the equation into

$$
\begin{gathered}
-\propto a^{3}+\frac{n^{2} c^{3}}{\propto^{2}}-a^{3}+\frac{a^{3}}{(1+\propto)^{2}}=0, \text { or } \\
\frac{n^{2} c^{3}}{a^{3}}-\propto^{2}(1+\propto)+\frac{\propto^{2}}{(1+\propto)^{2}}=0
\end{gathered}
$$

and once put, for conciseness, $\frac{n^{2} c^{3}}{a^{3}}=m$, gives

$$
m(1+\alpha)^{2}=\alpha^{2}(1-\alpha)^{3}-\alpha^{2}
$$

which is obtained from the equation of the same form above by taking $m$ and $\alpha$ negative.

Henceforth

$$
\propto=\sqrt[3]{\frac{m}{3}}+\frac{1}{3} \sqrt[3]{\frac{m^{2}}{9}}-\frac{1}{27} m-\frac{1}{81} m \sqrt[3]{\frac{m}{3}},
$$

however, as a first approximation $\propto=\sqrt[3]{\frac{m}{3}}$ and $v=\frac{c u}{a} \sqrt[3]{\frac{n^{2}}{3}}$ as before.

10) These cases are most worthy to be commented, since they could be worked out absolutely without any approximation, even if both forces of the Sun and of the Earth concur in producing motion, since there is no other case which this can happen. However, the body would, in fact, move with such a simple motion, provided it would be at the assigned distance, and while it would appear from the Earth in conjunction or in opposition with the Sun, a motion of this 
type would be impressed, when it had began to advance in the same pace with the Earth in the ecliptic plane. However, on the other hand, if the impressed motion differs from this law, it would not, in fact, remain in continuous conjunction or in opposition with the Sun, but it would perform tiny excursions and, because of this, as almost oscillating. In the case where the motion had minimally differed from the formulation that was found, in the usual way, also by approximation, it will be possible to define such motion; in this case with the threshold of irregular motions, which still cannot be approached by any calculation, certainly it is seen with no lack of usefulness, if I will seek more carefully the nature of such motion.

11) However, although this investigation is by no means involved by trivial difficulties, however, our equations can be made conveniently easier to handle, when the distance $v$ is much smaller than $u$, when it is possible to produce a convenient approximation. Of course, because $z=\sqrt{u u-2 u v \cos \eta+v v}$, we have approximately $\frac{1}{z^{3}}=\frac{1}{u^{3}}+\frac{3 v \cos \eta}{u^{4}}-\frac{3 v^{2}}{2 u^{5}}+\frac{15 v^{2} \cos \eta^{2}}{2 u^{5}}$, and thus $1-\frac{u^{3}}{z^{3}}=-\frac{3 v}{u} \cos \eta-\frac{3 v^{2}}{2 u^{2}}\left(1-5 \cos \eta^{2}\right)$, from which our equations that were found for the motion of the Moon will transform into the following forms:

$$
\begin{aligned}
& \text { I) } \frac{2 \mathrm{~d} v \mathrm{~d} \phi+v \mathrm{dd} \phi}{\mathrm{d} \zeta^{2}}-\frac{3 a^{3} v}{u^{3}} \sin \eta \cos \eta-\frac{3 a^{3} v^{2}}{2 u^{4}} \sin \eta\left(1-5 \cos \eta^{2}\right)=0 \\
& \text { II) } \frac{\mathrm{dd} v-v \mathrm{~d} \phi^{2}}{\mathrm{~d} \zeta^{2}}+\frac{n^{2} c^{3}}{v^{2}}+\frac{a^{3} v}{z^{3}}\left(1-3 \cos \eta^{2}+\frac{3 a^{3} v^{2}}{2 u^{4}}\left(3 \cos \eta-5 \cos \eta^{3}\right)\right)=0
\end{aligned}
$$

Then, also, the calculation can be made easier, if we consider the mean motion of the Sun, then $u=a$, and $\theta=\zeta$, and thus $\eta=\phi-\zeta$, or $\phi=\eta+\zeta$, whence arising the following equations

$$
\begin{aligned}
& \text { I) } \frac{2 \mathrm{~d} v \mathrm{~d} \eta+v \mathrm{dd} \eta}{\mathrm{d} \zeta^{2}}+\frac{2 \mathrm{~d} v}{\mathrm{~d} \zeta}+3 v \sin \eta \cos \eta-\frac{3 v^{2}}{2 a} \sin \eta\left(1-5 \cos \eta^{2}\right)=0 \\
& \text { II) } \frac{\mathrm{dd} v}{\mathrm{~d} \zeta^{2}}-v\left(1+\frac{\mathrm{d} \eta}{\mathrm{d} \zeta}\right)^{2}+v\left(1-3 \cos \eta^{2}\right)+\frac{n^{2} c^{3}}{v^{2}}+\frac{3 v^{2}}{2 a} \cos \eta\left(3-5 \cos \eta^{2}\right)=0
\end{aligned}
$$

where the last terms in these expressions can be omitted, since the fraction $\frac{v}{a}$ is very small, even establishing that the distance of the Moon is four times larger.

12) Now, be reminded the case where the Moon will be seen hesitating in almost oscillating motion around the Sun, and let us assume that the angle $\eta$ is as small as possible, such that $\sin \eta=\eta$, and $\cos \eta=1-\frac{1}{2} \eta^{2}$, and then we have:

I) $\frac{2 \mathrm{~d} v \mathrm{~d} \eta+v \mathrm{dd} \eta}{\mathrm{d} \zeta^{2}}+\frac{2 \mathrm{~d} v}{\mathrm{~d} \zeta}+3 v \eta=0$

II) $\frac{\mathrm{dd} v}{\mathrm{~d} \zeta^{2}}-v\left(1+\frac{\mathrm{d} \eta}{\mathrm{d} \zeta}\right)^{2}+\frac{n^{2} c^{3}}{v^{2}}-2 v+3 v \eta^{2}=0$

Then, because the distance $v$ is little changed, let us put $v=b(1+x)$, such that $x$ is a small quantity, and further, be for brevity $\frac{n^{2} c^{3}}{b^{3}}=m$, and hence 
I) $\frac{2 \mathrm{~d} x \mathrm{~d} \eta+x \mathrm{dd} \eta}{\mathrm{d} \zeta^{2}}+\frac{\mathrm{dd} \eta}{\mathrm{d} \zeta^{2}}+\frac{2 \mathrm{~d} x}{\mathrm{~d} \zeta}+3 \eta+3 x \eta=0$

II) $\frac{\mathrm{dd} x}{\mathrm{~d} \zeta^{2}}-3-3 x-\frac{2 \mathrm{~d} \eta}{\mathrm{d} \zeta}-\frac{2 x \mathrm{~d} \eta}{\mathrm{d} \zeta}-\frac{\mathrm{d} \eta^{2}}{\mathrm{~d} \zeta^{2}}-\frac{x \mathrm{~d} \eta^{2}}{\mathrm{~d} \zeta^{2}}+3 \eta^{2}+3 x \eta^{2}+m$

$$
-2 m x+3 m x^{2}=0
$$

whence, it is necessary to define the values of the quantities $x$ and $\eta$ for every angle $\zeta$.

13) Since the angle $\eta$ is minimum, and which alternates between negative and positive values, as the Moon is seen passing to and fro the Sun: it is easily allowed to conceive the existence of a relation between a certain angle $\omega$ and $\zeta$, and thus to define the following

$$
\eta=A \sin \omega+B \sin 2 \omega+C \sin 3 \omega \text { etc. }^{14}
$$

and also $\mathrm{d} \omega=\alpha \mathrm{d} \zeta$. Then,

$$
\begin{aligned}
& \frac{\mathrm{d} \eta}{\mathrm{d} \zeta}=\alpha A \cos \omega+2 \alpha B \cos 2 \omega+3 \alpha C \cos 3 \omega, \text { and } \\
& \frac{\mathrm{dd} \eta}{\mathrm{d} \zeta^{2}}=-\alpha^{2} A \sin \omega-4 \alpha^{2} B \sin 2 \omega-9 \alpha^{2} C \sin 3 \omega .
\end{aligned}
$$

On account that the first equation can be transformed into

$$
\frac{2 \mathrm{~d} x}{1+x}+\frac{\mathrm{dd} \eta+3 \eta \mathrm{d} \zeta^{2}}{\mathrm{~d} \eta+\mathrm{d} \zeta}=0
$$

which, upon integration gives

$$
2 \ln (1+x)+\ln \left(1+\frac{\mathrm{d} \eta}{\mathrm{d} \zeta}\right)+3 \int \frac{\eta \mathrm{d} \zeta}{1+\frac{\mathrm{d} \eta}{\mathrm{d} \zeta}}=\text { Const. }
$$

or, because $x$ and $\frac{\mathrm{d} \eta}{\mathrm{d} \zeta}$ are small quantities, then:

$$
\begin{aligned}
& 2 x-x^{2}+\frac{2}{3} x^{3}+\frac{\mathrm{d} \eta}{\mathrm{d} \zeta}-\frac{\mathrm{d} \eta^{2}}{2 \mathrm{~d} \zeta^{2}}+\frac{\mathrm{d} \eta^{3}}{3 \mathrm{~d} \zeta^{3}}+3 \int \eta \mathrm{d} \zeta-\frac{3}{2} \eta^{2} \\
& +3 \int \frac{\eta \mathrm{d} \eta^{2}}{\mathrm{~d} \zeta}-3 \int \frac{\eta \mathrm{d} \eta^{3}}{\mathrm{~d} \zeta^{2}}=\text { Const. }
\end{aligned}
$$

Now, since $\mathrm{d} \zeta=\frac{\mathrm{d} \omega}{\alpha}$, then

$$
\int \eta \mathrm{d} \zeta=-\frac{A}{\alpha} \cos \omega-\frac{B}{2 \alpha} \cos 2 \omega-\frac{C}{3 \alpha} \cos 3 \omega
$$

${ }^{14}$ This appears to be an archaic form of a Fourier sine series expansion.

${ }^{15}$ These integrals are the result of a series expansion of $\frac{1}{1+\frac{\mathrm{d} \eta}{\mathrm{d} \zeta}}=1-\frac{\mathrm{d} \eta}{\mathrm{d} \zeta}+\frac{\mathrm{d} \eta^{2}}{\mathrm{~d} \zeta^{2}}-\frac{\mathrm{d} \eta^{3}}{\mathrm{~d} \zeta^{3}}$, and then, $3 \int \frac{\eta \mathrm{d} \zeta}{1+\frac{\mathrm{d} \eta}{\mathrm{d} \zeta}}=3 \int \eta \mathrm{d} \zeta-3 \int \eta \mathrm{d} \eta+3 \int \frac{\eta \mathrm{d} \eta^{2}}{\mathrm{~d} \zeta}-3 \int \frac{\eta \mathrm{d} \eta^{3}}{\mathrm{~d} \zeta^{2}}$ 


$$
\begin{gathered}
\eta^{2}=\frac{1}{2} A^{2}+A B \cos \omega-\frac{1}{2} A^{2} \cos 2 \omega-A B \cos 3 \omega \\
+\frac{1}{2} B^{2} \quad+A C \\
\frac{\mathrm{d} \eta^{2}}{\mathrm{~d} \zeta^{2}}=\frac{1}{2} \alpha^{2} A^{2}+2 \alpha^{2} A B \cos \omega+\frac{1}{2} \alpha^{2} A^{2} \cos 2 \omega+2 \alpha^{2} A B \cos 3 \omega \\
+2 \alpha^{2} B^{2} \quad+3 \alpha^{2} A C \\
\frac{\mathrm{d} \eta^{3}}{\mathrm{~d} \zeta^{3}}=\alpha^{3} A^{2} B+\frac{3}{4} \alpha^{3} A^{3} \cos \omega+\alpha^{3} A^{2} B \cos 2 \omega \\
+4 \alpha^{3} A B^{2}
\end{gathered}
$$

where we justly disregarded higher powers of the letters $A, B, C$.

14) Since

$$
\begin{aligned}
\frac{\eta \mathrm{d} \eta^{2}}{\mathrm{~d} \zeta^{2}}= & \frac{1}{4} \alpha^{2} A^{3} \sin \omega+\frac{3}{2} \alpha^{2} A^{2} B \sin 2 \omega & +\frac{1}{4} \alpha^{2} A^{3} \sin 3 \omega \\
& +3 \alpha^{2} A B^{2}+2 \alpha^{2} B^{3} & +\frac{3}{2} \alpha^{2} A^{2} C \\
& -\frac{3}{2} \alpha^{2} A^{2} C & +\alpha^{2} A B^{2}
\end{aligned}
$$

and because $\mathrm{d} \zeta=\frac{\mathrm{d} \omega}{\alpha}$, then

$$
\begin{array}{rlr}
\int \frac{\eta \mathrm{d} \eta^{2}}{\mathrm{~d} \zeta}= & -\frac{1}{4} \alpha A^{3} \cos \omega-\frac{3}{4} \alpha A^{2} B \cos 2 \omega & -\frac{1}{12} \alpha A^{3} \cos 3 \omega \\
& -3 \alpha A B^{2} \quad-\alpha B^{3} & -\frac{1}{2} \alpha A^{2} C \\
& +\frac{3}{2} \alpha A^{2} C & -\frac{1}{2} \alpha A B^{2}
\end{array}
$$

where, since the series $A, B, C$ had already decreased very much, further terms can be omitted. Then, since

$$
\frac{\eta \mathrm{d} \eta^{3}}{\mathrm{~d} \zeta^{3}}=\frac{7}{8} \alpha^{3} A^{3} B \sin \omega+\frac{3}{8} \alpha^{3} A^{4} \sin 2 \omega+\frac{7}{8} \alpha^{3} A^{3} B \sin 3 \omega
$$

which upon integration gives

$$
\int \frac{\eta \mathrm{d} \eta^{3}}{\mathrm{~d} \zeta^{2}}=-\frac{7}{8} \alpha^{3} A^{3} B \cos \omega-\frac{3}{16} \alpha^{3} A^{4} \cos 2 \omega-\frac{7}{24} \alpha^{3} A^{3} B \cos 3 \omega,
$$

and finally, from the expressions above, and omitting the terms that are constants, the following equation is obtained

$$
\begin{gathered}
2 x-x^{2}+\frac{2}{3} x^{3}+\alpha A \cos \omega+2 \alpha B \cos 2 \omega+3 \alpha C \cos 3 \omega=0^{16} \\
-\alpha^{2} A B \quad-\frac{1}{4} \alpha^{2} A^{2} \quad-\alpha^{2} A B
\end{gathered}
$$

${ }^{16}$ The expanded forms of the integrals $3 \int \frac{\eta \mathrm{d} \eta^{2}}{\mathrm{~d} \zeta}-3 \int \frac{\eta \mathrm{d} \eta^{3}}{\mathrm{~d} \zeta^{2}}$ were not included in this equation. 


$$
\begin{array}{lll}
+\frac{1}{4} \alpha^{3} A^{3} & -\frac{3}{2} \alpha^{2} A C & -\frac{C}{\alpha} \\
-\frac{3 A}{\alpha} & +\frac{1}{3} \alpha^{3} A^{2} B & +\frac{3}{2} A B \\
-\frac{3}{2} A B & -\frac{3 B}{2 \alpha} & -\frac{1}{4} \alpha A^{3} \\
-\frac{3}{4} \alpha A^{3} & +\frac{3}{4} A^{2} & +\frac{1}{4} \alpha^{3} A^{3} \\
& -\frac{3}{2} A C & \\
-\frac{9}{4} \alpha A^{2} B &
\end{array}
$$

15) To find the value of $x$, let us put for conciseness

$$
\begin{gathered}
\left(\alpha-\frac{3}{\alpha}\right) A-\left(\alpha^{2}+\frac{3}{2}\right) A B+\frac{1}{4} \alpha\left(\alpha^{2}-3\right) A^{3}=\mathfrak{A} \\
\frac{4 \alpha^{2}-3}{2 \alpha} B-\frac{\left(\alpha^{2}-3\right)}{4} A^{2}=\mathfrak{B} \\
\frac{3 \alpha^{2}-1}{\alpha} C-\frac{\left(2 \alpha^{2}-3\right)}{2} A B+\frac{1}{4} \alpha\left(\alpha^{2}-1\right) A^{3}=\mathfrak{C}
\end{gathered}
$$

such that

$$
\begin{gathered}
2 \ln (1+x)+\mathfrak{A} \cos \omega+\mathfrak{B} \cos 2 \omega+\mathfrak{C} \cos 3 \omega=0 \\
1+x=\mathrm{e}^{-\frac{1}{2} \mathfrak{A} \cos \omega-\frac{1}{2} \mathfrak{B} \cos 2 \omega-\frac{1}{2} \mathfrak{C} \cos 3 \omega},
\end{gathered}
$$

whence, we conclude that

$$
\begin{aligned}
& x=-\frac{1}{2} \mathfrak{A} \cos \omega-\frac{1}{2} \mathfrak{B} \cos 2 \omega-\frac{1}{2} \mathfrak{C} \cos 3 \omega \\
& +\frac{1}{8} \mathfrak{A} \mathfrak{B} \quad+\frac{1}{16} \mathfrak{A}^{2} \quad+\frac{1}{8} \mathfrak{A} \mathfrak{B} \\
& +\frac{1}{64} \mathfrak{A}^{3} \quad-\frac{1}{192} \mathfrak{A}^{3}
\end{aligned}
$$

But for us not to be involved in excessively tedious calculations, we shall procure a less accurate expression, by neglecting the triple angle, such that $\eta=A \sin \omega+B \sin 2 \omega$, and then we have

$$
\begin{aligned}
x=-\frac{\left(\alpha^{2}-3\right)}{2 \alpha} A \cos \omega & -\frac{\left(4 \alpha^{2}-3\right)}{4 \alpha} B \cos 2 \omega \\
& +\frac{3\left(\alpha^{2}-1\right)\left(\alpha^{2}-3\right)}{16 \alpha^{2}} A^{2}
\end{aligned}
$$

where, for conciseness, we put

$$
x=E \cos \omega+F \cos 2 \omega
$$

such that

$$
E=\frac{3-\alpha^{2}}{2 \alpha} A \text { and } F=\frac{3-4 \alpha^{2}}{4 \alpha} B+\frac{3\left(\alpha^{2}-1\right)\left(\alpha^{2}-3\right)}{16 \alpha^{2}} A^{2}
$$


16) When these values are substituted in the second equation, we will find out that $^{17}$ :

$$
\begin{aligned}
& \frac{\mathrm{d} d x}{\mathrm{~d} \zeta^{2}}=\quad-\alpha^{2} E \cos \omega-4 \alpha^{2} F \cos 2 \omega \\
& -3-3 x=-3 \quad-3 E \quad-3 F \\
& -\frac{2 \mathrm{~d} \eta}{\mathrm{d} \zeta}=-\alpha A E-2 \alpha A \quad-4 \alpha B \\
& -2 \alpha B E-\alpha A E \\
& -\frac{2 x \mathrm{~d} \eta}{\mathrm{d} \zeta}=\quad-\alpha \mathrm{AF} \\
& -\frac{\mathrm{d} \eta^{2}}{\mathrm{~d} \zeta^{2}}=-\frac{1}{2} \alpha^{2} A^{2}+2 \alpha^{2} A B \quad-\frac{1}{2} \alpha^{2} A^{2} \\
& +3 \eta^{2}=+\frac{3}{2} A^{2}+3 A B \cos \omega-\frac{3}{2} A^{2} \cos 2 \omega \\
& +m=+m \\
& +2 m x=\quad-2 m E-2 m F \\
& +3 m x^{2}=+\frac{3}{2} m E^{2}+3 m E F \quad+\frac{3}{2} m E^{2}
\end{aligned}
$$

whence, we first conclude that ${ }^{18}$ :

$$
m\left(1+\frac{3}{2} E^{2}\right)=3+\alpha A E+\frac{1}{2} \alpha^{2} A^{2}-\frac{3}{2} A^{2}=3
$$

thence

$$
m=3-\frac{9}{2} E^{2}
$$

for the determination of the number $m$ and then the distance $b$.

It is clear that $m=3$, approximately, and thus $b=c \sqrt[3]{\frac{n^{2}}{3}}$. We have further that ${ }^{19}$

$$
-\alpha^{2} E-9 E-2 \alpha A-2 \alpha B E-\alpha A F\left(2 \alpha^{2}+3\right) A B+9 E F=0
$$

which, by neglecting terms which cannot be simply reduced to $\frac{E}{A}=\frac{3-\alpha^{2}}{2 \alpha}$, gives

$$
\begin{gathered}
\left(\alpha^{2}+9\right)\left(3-\alpha^{2}\right)+4 \alpha^{2}=0, \text { or } \\
\alpha^{4}+2 \alpha^{2}-27=0 \text {, and then, } \alpha^{2}=\sqrt{28}-1 .
\end{gathered}
$$

Finally, the third equation gives ${ }^{20}$

$$
-\left(4 \alpha^{2}+9\right) F-4 \alpha B-\frac{3}{2} A^{2} A E-\frac{1}{2} \alpha^{2} A^{2}-\frac{3}{2} A^{2}+\frac{9}{2} E^{2}=0,
$$

${ }^{17}$ The reduced form of $-\frac{x \mathrm{~d} \eta^{2}}{\mathrm{~d} \zeta^{2}}$ from the second equation was not included in the calculations.

${ }^{18}$ The following expression is the result of equating the constant terms to zero.

${ }^{19}$ The following expression is the result of equating the coefficients of $\cos \omega$ to zero.

${ }^{20} \mathrm{The}$ following expression is the result of equating the coefficients of $\cos 2 \omega$ to zero. 
therefore:

$$
\left.\begin{array}{c}
\frac{\left(4 \alpha^{2}-3\right)\left(4 \alpha^{2}+9\right)}{4 \alpha} B-\frac{3\left(\alpha^{2}-1\right)\left(\alpha^{2}-3\right)\left(4 \alpha^{2}+9\right)}{16 \alpha^{2}} A^{2} \\
-4 \alpha B \quad \frac{\alpha^{2}-3}{2} A^{2} \\
-\frac{\left(\alpha^{2}+3\right)}{2} A^{2} \\
+\frac{9\left(\alpha^{2}-3\right)^{2}}{8 \alpha^{2}} A^{2}
\end{array}\right\}
$$

which, after rearrangement gives:

$$
B\left(16 \alpha^{4}+8 \alpha^{2}-27\right)=\frac{3}{2} A^{2} \alpha\left(13-7 \alpha^{2}+\frac{3 A^{2}}{2 \alpha}\right),
$$

or since $27=\alpha^{4}+2 \alpha^{2}$, then

$$
3 B\left(5 \alpha^{2}+2\right)=\frac{3 A^{2}}{2 \alpha}\left(13-7 \alpha^{2}+2 \alpha^{4}\right),
$$

and, therefore,

$$
\begin{gathered}
B=\frac{13-7 \alpha^{2}+2 \alpha^{4}}{2 \alpha\left(5 \alpha^{2}+2\right)} A^{2}=\frac{67-11 \alpha^{2}}{2 \alpha\left(5 \alpha^{2}+2\right)} A^{2} \\
F=\frac{291-94 \alpha^{2}-23 \alpha^{4}}{8 \alpha^{2}\left(5 \alpha^{2}+2\right)} A^{2}=-\frac{165-24 \alpha^{2}}{4 \alpha^{2}\left(5 \alpha^{2}+2\right)} A^{2}
\end{gathered}
$$

Then, accordingly, the value of $A$ is at our discretion, which depends on the digressions from the syzygy line $e^{21}$, which it is proper to assume as being a very small fraction, such that the quadratic terms can be considered of second order, being sufficient only the first terms. Then, for the distance $v=b(1+x)$, we have that $b=c \sqrt[3]{\frac{n^{2}}{3}}$, and the angle $\omega$ is defined such that $\omega=\alpha \zeta+\beta$, and since $\alpha^{2}=\sqrt{28}-1$, then, $\alpha^{2}=4.291502$ and $\alpha=2.071594$. Thereupon, putting $\eta=A \sin \omega$ and $v=b\left(1-\frac{\left(\alpha^{2}-3\right)}{2 \alpha} A \cos \omega\right)$ or $v=b(1-0.311717 A \cos \omega)$.

The excursions are maximum for angles $\omega$ equal to $90^{\circ}, 270^{\circ}$, etc., therefore, between one maximum digression to the next we have $\alpha \zeta=180^{\circ}$ and $\zeta=86^{\circ} 53.5^{\prime}$ : with the greatest of these digressions given by $v=b$. But in case this kind of libration ${ }^{22}$ happens to be greater, its determination involves consi-

\footnotetext{
${ }^{21} \mathrm{~A}$ kind of unity, namely an alignment of three celestial bodies (for example, the Sun, Earth, and Moon) such that one body is directly between the other two, such as occurs at an eclipse (from the Wikipedia).

${ }^{22}$ Is the wagging of the Moon perceived by Earth-bound observers caused by changes in their perspective. It permits an observer to see slightly different halves of the surface at different times. It is similar in both cause and effect to the changes in the Moon's apparent size due to changes in distance (from the Wikipedia).
} 
derable difficulties, because, as more accurately we wish to define all the variations, less certain we would be about the remaining that we have overlooked.

\section{An Easy Method for Calculating the Motion of Celestial Bodies Perturbed in Any Manner Avoiding Astronomical Computations (Methodus Facilis Motus Corporum Coelestium Utcunque Perturbatos Ad Rationem Calculi Astronomici Revocandi, Euler, 1768)}

I) Although I have often attacked the investigation on how the motions of celestial bodies are perturbed due to their mutual action, most of the time, I have incurred in rather lengthy and laborious calculations, which, however, after many digressions, could be reduced to simpler formulas. However, the cause of this prolixity is due to a multiplicity of elements, which are necessary to introduce into the calculation, that is: not only for all the determinations, which are related to the motion of the perturbing bodies, should be examined, but also the perturbation of its own motion, in so far as if it is not in the same plane, it demands various elements, to which is a custom among Astronomers to consider variations originated in the nodal line ${ }^{23}$ and inclination of the orbit. But in case all these considerations are simultaneously included into the calculation, it is really not worthwhile, because they will give rise to much trouble and confusion, to which no other remedy is seen to exist, unless all the elements are carefully distinguished, and all the operations are established so that no more elements are admitted into them, than those that are necessary to consider.

II) The principal part of this research is related to mechanics, when the perturbation of the motion by the forces of the disturbing body should be defined; thus mechanical principles are provided, and from them the location of the body, whose motion is seeked, can be conveniently determined at any time by three mutually orthogonal coordinates; truly the other part scarcely requires a swifter development, with which the location is firstly determined, and should be reduced to the acceptable practice in Astronomy, in which it is common to naturally express the different locations in the sky by longitude and latitude. And also in this second part, in which will be allowed to recall the geometry, being correct to distinguish a priori, that all mechanics is due to it, and I should observe that these two parts can furthermore not only be conveniently separated, but also to be able to deal with both matters in a much easier way than if we wished to deal jointly with both of them. However, it is seen that the geometrical mechanical investigation should precede, nonetheless it is possible to begin with the geometrical part in a neat way, with none impediment, from the location of the body, which motion we seek to obtain, as it were known, and that we identify by three coordinates. This inversion of the methodology is thus seen to follow, so that the development of the geometrical part gives much important support, with which the work in the calculation of the mechanical part that follows next will be con-

\footnotetext{
${ }^{23}$ The nodal line is a line that joins the ascending node and the descending node of an orbit. It marks the intersection of the orbital plane and some reference plane, usually the ecliptic.
} 
siderably alleviated. We follow this method certainly with great advantage, provided that we handle the geometrical part without introducing into the calculation quantities related to the disturbing forces.

\section{GEOMETRICAL PART}

III) Therefore, I assume that the motion of the body $Z$ is to be determined and that, as usual, readily defined by mechanical principles. Certainly, firstly the motion in relation to certain point $A$, which is considered fixed, even if it happened that the said point is used as reference to the circular motion, then, next, a certain plane is considered traversing through this point and equally fixed, which is represented by the plane of the figure itself, in which it is drawn a fixed line $A B$, and at any time, the location of the body $Z$ is thus defined by the three mutually orthogonal coordinates $A X, X Y$ and $Y Z$, so that first, from the $Z$ location, the perpendicular $Z Y$ to that plane is drawn, further on, truly from $Y$, the normal $Y X$ is guided in the direction of the line $A B$. Then, let us call the following three coordinates:

$$
A X=X, X Y=Y \text { and } Y Z=Z
$$

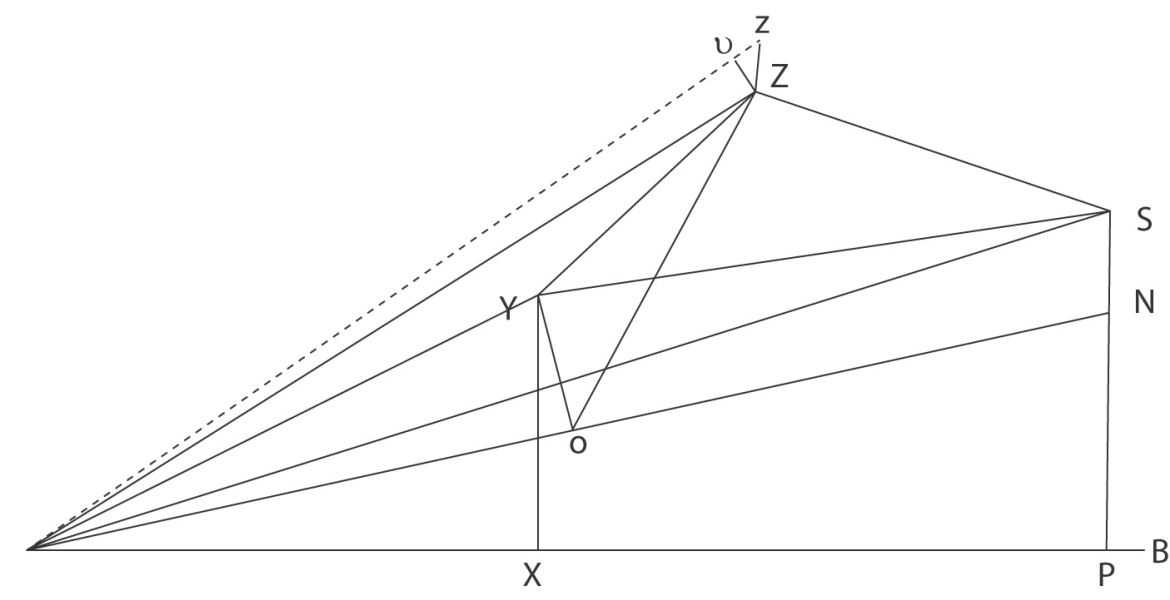

which values at any elapsed time $=t$ are considered to be known. Then, consequently, the distance of the body $Z$ to the fixed point $A$ is immediately obtained, which, for brevity, it is indicated by $A Z=v$, and then, $v^{2}=X^{2}+Y^{2}+Z^{2}$.

Then, we conceive that during the time $\mathrm{d} t$ the body advances from $Z$ to $Z$, such that $A z=v+\mathrm{d} v$, and the elementary angle $Z A Z=\mathrm{d} \varnothing$, which, in the mean time, the body $Z$ is seen to complete in its orbit around $A$, resulting in $Z z=\sqrt{\mathrm{d} v^{2}+v^{2} \mathrm{~d} \varnothing^{2}}$, whereas, according to the coordinate elements we have that $\mathrm{Zz}=\sqrt{\mathrm{d} X^{2}+\mathrm{d} Y^{2}+\mathrm{d} Z^{2}}$, whence

$$
\mathrm{d} v^{2}+v^{2} \mathrm{~d} \varnothing^{2}=\mathrm{d} X^{2}+\mathrm{d} Y^{2}+\mathrm{d} Z^{2}
$$

in this way, this exposes how the elementary angle $\mathrm{d} \varnothing$ can be conveniently expressed by the coordinates, which will be soon succinctly shown.

IV) A certain plane is defined by the segment $Z z$ and point $A$, in which the body $Z$ is, in fact, considered to move: this plane will cut somewhere the fixed plane of 
the figure; then this intersection is constructed along the line $A N$, which is called the nodal line in Astronomy, and which variation, due to the motion perturbation of the body $Z$, should be investigated above all: next, it is also convenient to note, that the angle which the plane $N A Z$ is inclined towards the fixed plane, which in Astronomy is simply called inclination, and because of the perturbation of the motion, can undergo remarkable alterations. Then, next, let us consider these new elements:

$$
\begin{aligned}
& \text { Longitude of the nodal line or angle } B A N=\psi \\
& \text { Inclination of the orbit to the fixed plane }=\omega \\
& \text { and argument of latitude or angle } N A Z=\sigma
\end{aligned}
$$

which we rename according to the coordinates, so from $Y$, as well as from $Z$, let us draw the normals $Y O$ and $Z O$ to the nodal line $A N$, such that the angle $Y O Z$ will have the same inclination of $\omega$.

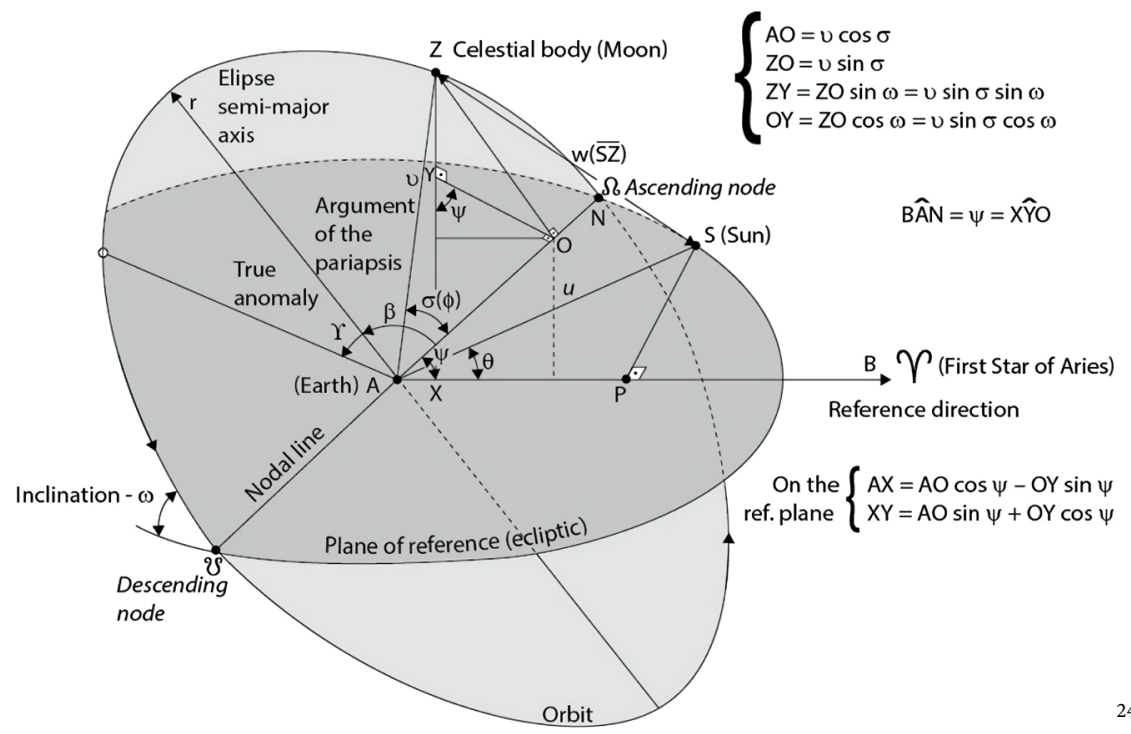

Then, considering that the angle $N A Z=\sigma$, and that the distance $A Z=v$, we will have that:

$$
A O=v \cos \sigma \text { and } Z O=v \sin \sigma
$$

and further

$$
Z Y=v \sin \sigma \sin \omega \text { and } O Y=v \sin \sigma \cos \omega
$$

and since the angle $B A N=\psi=X Y O$, we conclude that:

$$
A X=v \cos \sigma \cos \psi-v \sin \sigma \cos \omega \sin \psi
$$

and $A Y=v \cos \sigma \sin \psi+v \sin \sigma \cos \omega \cos \psi$.

Therefore, our three coordinates are thus defined:

$$
\begin{aligned}
& X=v(\cos \sigma \cos \psi-\sin \sigma \cos \omega \sin \psi) \\
& Y=v(\cos \sigma \sin \psi+\sin \sigma \cos \omega \cos \psi)
\end{aligned}
$$

${ }^{24}$ This figure was added by the Translator. 


$$
\text { and } Z=v \sin \sigma \sin \omega,
$$

where it should be noted that the tangent of the angle $N A Y=\tan \sigma \cos \omega$, which is an angle called the longitude of the point $Z$ to the node.

V) Since in Astronomy the angle $B A Y$ reveals the longitude, truly the angle $Z A Y$ is the latitude of point $Z$, which relates to the plane of the ecliptic, and the line $A B$ extended to the First Star of Aries; each one of these denominations can be used in a wider sense: we then have

longitude of the point $Z$ or angle $B A Y=\psi+N A Y$

considering that the tangent of the angle $N A Y=\tan \sigma \cos \omega$

for the latitude or truly for the angle $Z A Y$ we will have

$$
\sin Z A Y=\frac{Z Y}{A Z}=\sin \sigma \sin \omega
$$

where the same formulas are usually obtained from the spherical trigonometry. Certainly, in the spherical surface with center in $A$, the maximum circle $B N Y$ represents a fixed plane, and the point $B$ at infinite, and from which the longitude is calculated. Furthermore, be $N$ the node and $N Z$ the orbit to which now the motion of the body $Z$ is referred to, then, from $Z$, in the direction of the circle $B N Y$, the normal arch $Z Y$ is drawn; once this is done, the arch $B N Y$ shows the longitude, and truly, the arch $Z Y$ the latitude of the point $Z$, and in relation to these we have that:

$\operatorname{arch} B N$, or angle of the node $=\psi$

angle $Z N Y$ or inclination $=\omega$

and $\operatorname{arch} N Z$ or argument of the latitude $=\sigma$

from these, the solution of the right spherical triangle $N Y Z$ gives

$$
\sin Z Y=\sin \sigma \sin \omega \text { and } \tan N Y=\tan \sigma \cos \omega,
$$

exactly as before.

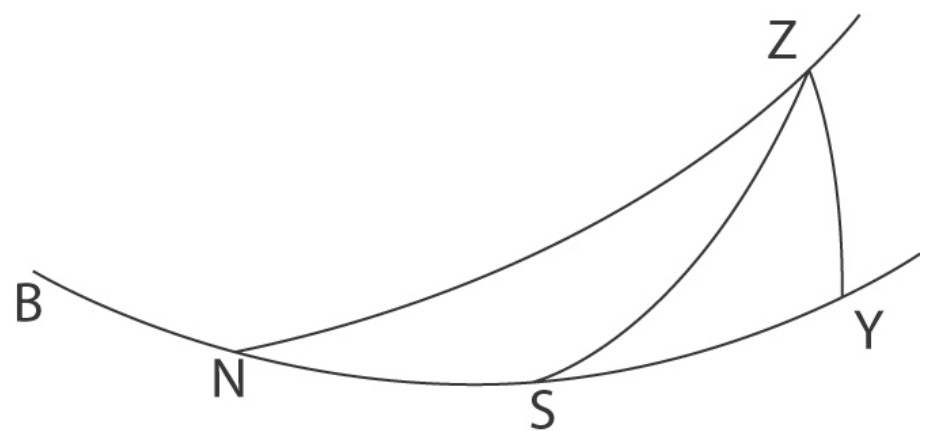

VI) However, for the nodal line and the inclination be both variables; since both the point $Z$ and $z$ belong to the same plane $N A Z$, then by differentiation, the point $\mathrm{Z}$ should come to $\mathrm{z}$, and because the angle $B A N=\psi$ and the inclination $\omega$ are considered constants, of course as long as the angle $N A Z=\sigma$, the elementary angle $Z A z=\mathrm{d} \varnothing$ is assumed to increase, such that $\mathrm{d} \sigma=\mathrm{d} \varnothing$. However, to 
came to the same point $z$ in another way, it is necessary that the nodal line and the inclination be both considered variables, since the point $z$ should also have a tendency to a diversified orbit, and then, the differential $\mathrm{d} \sigma$ should not be considered to be equal to $\mathrm{d} \varnothing$ itself, but it should attain its proper value, which at the same time depends on the variation of the orbit. Therefore, since this double differentiation should lead to the same equations that we will obtain next, for which certain relations between the variations originated in the orbit will be defined, which will provide a maximum usage in a subsequent calculation. In fact, it possesses not only the differentiations of the local coordinates themselves, but also of the quantities thence derived, such as:

$$
\frac{X}{Z}=\frac{\cos \sigma \cos \psi}{\sin \sigma \sin \omega}-\frac{\cos \omega \sin \psi}{\sin \omega} \text { and } \frac{Y}{Z}=\frac{\cos \sigma \sin \psi}{\sin \sigma \sin \omega}+\frac{\cos \omega \cos \psi}{\sin \omega}
$$

which ought, therefore, to provide the same results obtained in both ways for the double differentials.

VII) Therefore, firstly assuming that the angles $\psi$ and $\omega$ are constants and that $\mathrm{d} \sigma=\mathrm{d} \varnothing$, the differentials are:

$$
\mathrm{d}\left(\frac{X}{Z}\right)=\frac{-\mathrm{d} \varnothing \cos \psi}{\sin ^{2} \sigma \sin \omega} \text { and } \mathrm{d}\left(\frac{Y}{Z}\right)=\frac{-\mathrm{d} \varnothing \sin \psi}{\sin ^{2} \sigma \sin \omega} \text {. }
$$

For the other differentiation [considering that $\psi$ and $\omega$ are also variables], it should be firstly noted that:

$$
\frac{X}{Z} \cos \psi+\frac{Y}{Z} \sin \psi=\frac{\cos \sigma}{\sin \sigma \sin \omega} \text { and } \frac{Y}{Z} \cos \psi-\frac{X}{Z} \sin \psi=\frac{\cos \omega}{\sin \omega}
$$

which, in the usual way of differentiating gives:

$$
\begin{gathered}
\cos \psi \mathrm{d}\left(\frac{X}{Z}\right)+\sin \psi \mathrm{d}\left(\frac{Y}{Z}\right)+\mathrm{d} \psi\left(\frac{Y}{Z} \cos \psi-\frac{X}{Z} \sin \psi\right) \\
=\frac{-\mathrm{d} \sigma}{\sin ^{2} \sigma \sin \omega}-\frac{\mathrm{d} \omega \cos \sigma \cos \omega}{\sin \sigma \sin ^{2} \omega} \\
\cos \psi \mathrm{d}\left(\frac{Y}{Z}\right)-\sin \psi \mathrm{d}\left(\frac{X}{Z}\right)-\mathrm{d} \psi\left(\frac{X}{Z} \cos \psi+\frac{Y}{Z} \sin \psi\right)=\frac{-\mathrm{d} \omega}{\sin ^{2} \omega}
\end{gathered}
$$

whence, when the former values are substituted results in $^{25}$ :

$$
\begin{gathered}
\frac{-\mathrm{d} \varnothing \cos ^{2} \psi}{\sin ^{2} \sigma \sin \omega}-\frac{\mathrm{d} \varnothing \sin ^{2} \psi}{\sin ^{2} \sigma \sin \omega}+\frac{\mathrm{d} \psi \cos \omega}{\sin \omega}=\frac{-\mathrm{d} \sigma}{\sin ^{2} \sigma \sin \omega}-\frac{\mathrm{d} \omega \cos \sigma \cos \omega}{\sin \sigma \sin ^{2} \omega} \\
-\frac{\mathrm{d} \varnothing \sin \psi \cos \psi}{\sin ^{2} \sigma \sin \omega}+\frac{\mathrm{d} \varnothing \sin \psi \cos \psi}{\sin ^{2} \sigma \sin \omega}-\frac{\mathrm{d} \psi \cos \sigma}{\sin \sigma \sin \omega}=\frac{-\mathrm{d} \omega}{\sin ^{2} \omega}
\end{gathered}
$$

which are reduced into these:

$$
\begin{gathered}
-\frac{\mathrm{d} \varnothing}{\sin ^{2} \sigma \sin \omega}+\frac{\mathrm{d} \psi \cos \omega}{\sin \omega}=\frac{-\mathrm{d} \sigma}{\sin ^{2} \sigma \sin \omega}-\frac{\mathrm{d} \omega \cos \sigma \cos \omega}{\sin \sigma \sin ^{2} \omega} \\
-\frac{\mathrm{d} \psi \cos \sigma}{\sin \sigma \sin \omega}=\frac{-\mathrm{d} \omega}{\sin ^{2} \omega} \text { or } \frac{\mathrm{d} \omega}{\sin \omega}=\frac{\mathrm{d} \psi}{\tan \sigma}
\end{gathered}
$$

which when substituted into the previous expression gives

\footnotetext{
${ }^{25} \mathrm{No}$ justification is given by Euler for this equality.
} 


$$
\frac{\mathrm{d} \sigma-\mathrm{d} \varnothing}{\sin ^{2} \sigma \sin \omega}=\frac{-\mathrm{d} \psi \cos \omega}{\sin \omega}-\frac{\mathrm{d} \psi \cos ^{2} \sigma \cos \omega}{\sin ^{2} \sigma \sin \omega}=\frac{-\mathrm{d} \psi \cos \omega}{\sin ^{2} \sigma \sin \omega}
$$

or $\mathrm{d} \psi \cos \omega=\mathrm{d} \varnothing-\mathrm{d} \sigma$.

VIII) Whence, therefore, we first learned that the variation in the inclination of the orbit giving rise to $\mathrm{d} \omega$ is such that it always depends on the variation of the nodal line $\mathrm{d} \psi$ that is, $\mathrm{d} \omega=\frac{\mathrm{d} \psi \sin \omega}{\tan \sigma}$; or the increment in the inclination will be due to the promotion of the nodal line, as the sine of the inclination to the tangent of the argument of the latitude; whence the following consequences can be drawn from it:

1) If the argument of the latitude $\sigma$ is zero or 6 s where the latitude is zero, in the mean time the nodal line will tend to remain at rest the more the inclination is varied ${ }^{26}$.

2) If the argument of the latitude $\sigma$ is 3 s or 9 s where the latitude is zero, or $\tan \sigma=\infty$ where the latitude is maximum, then the inclination will not vary; regardless if in the mean time the nodal line progresses or regresses.

3) If the argument of the latitude $\sigma$ is contained between the limits 0 s and $3 \mathrm{~s}$ or between $6 \mathrm{~s}$ and $9 \mathrm{~s}$, that is, while the latitude increases, then the inclination $\omega$ increases, if indeed the nodal line advances, but if it retreats, the inclination diminishes.

4) If the argument of the latitude $\sigma$ is contained between the limits $3 \mathrm{~s}$ and $6 \mathrm{~s}$ or between $9 \mathrm{~s}$ and $12 \mathrm{~s}$, that is, while the latitude decreases, then the advancement of the nodal line inclination lessens, and in reality it ceases to be increased.

IX) Next, it should be observed that the increase of the argument of the latitude $\sigma$ promoted in its own orbit is not equal to the element $\mathrm{d} \varnothing$, unless the nodal line stays immovable; since we found that $\mathrm{d} \sigma=\mathrm{d} \varnothing-\mathrm{d} \psi \cos \omega$, with the exception in the case when the inclination $\omega$ were a right angle. These phenomena will be expressed more clearly by using the spherical trigonometry. If in fact, as before, the circle $B N Y$ represents a fixed plane, which the motion of the point $Z$ is referred to, so that its present motion takes place according to the circle $N Z$, such that $B N=\psi, Y N Z=\omega$ and the arch $N Z=\sigma$, whereas after the point $Z$ has progressed through the element $Z z=\mathrm{d} \varnothing$, and with its motion taking place according to the circle $n z$, the promotion of the nodal line will be given by $N n=\mathrm{d} \psi$, the inclination is transformed into $Y n z=\omega+\mathrm{d} \omega$, and the argument of the latitude into $n z=\sigma+\mathrm{d} \sigma$. Accordingly, the elemental arch $n v$ is drawn normal to $N Z$, and then we will have that $N v=\mathrm{d} \psi \cos \omega$ and $n v=\mathrm{d} \psi \sin \omega$; thence it is deduced that $Z n=\sigma-\mathrm{d} \psi \cos \omega$, and on this account $n z=\sigma-\mathrm{d} \psi \cos \omega+\mathrm{d} \varnothing=\sigma+\mathrm{d} \sigma$ and consequently, $\mathrm{d} \sigma=\mathrm{d} \varnothing-\mathrm{d} \psi \cos \omega$, as before; however, at the same time we see that because $\mathrm{d} \psi \cos \omega=\mathrm{d} \varnothing-\mathrm{d} \psi=N \nu$, the expression $\mathrm{d} \varnothing-\mathrm{d} \psi$ exhibits the promotion of the nodal line in the orbit itself, because since the node was in the point $N$ of the orbit $N Z$; it has now been transferred to the point $n$ or to $v$. In addition, from the spherical triangle $N n Z$, we have that:

\footnotetext{
${ }^{26}$ The ecliptic was sometimes divided into 12 signs, each subdivided into 30 degrees.
} 


$$
\sin \omega: \sin (\omega+\mathrm{d} \omega)=\sin (\sigma-\mathrm{d} \psi \cos \omega): \sin \sigma^{27}
$$

or

$$
\sin \omega: \sin \omega+\mathrm{d} \omega \cos \omega=\sin \sigma-\mathrm{d} \psi \cos \sigma \cos \omega: \sin \sigma
$$

and dividing by

$$
\sin \omega: \mathrm{d} \omega \cos \omega=\sin \sigma-\mathrm{d} \psi \cos \sigma \cos \omega: \mathrm{d} \psi \cos \sigma \cos \omega^{28}
$$

gives

$$
\mathrm{d} \omega \sin \sigma=\mathrm{d} \psi \sin \omega \cos \sigma^{29} \text { or } \frac{\mathrm{d} \omega}{\sin \omega}=\frac{\mathrm{d} \psi}{\tan \sigma}
$$

exactly as before ${ }^{30}$.

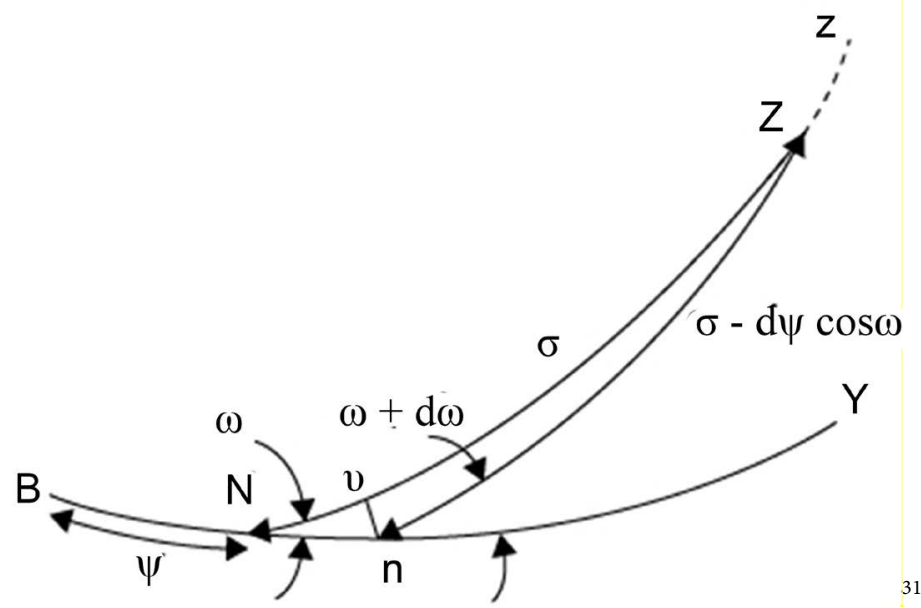

$\mathrm{X})$ Moreover, if the differential formulas found in $\$ 7$. are unfolded they give

$$
\frac{X \mathrm{~d} Z-Z \mathrm{~d} X}{Z^{2}}=\frac{\mathrm{d} \varnothing \cos \psi}{\sin ^{2} \sigma \sin \omega} \text { and } \frac{Y \mathrm{~d} Z-Z \mathrm{~d} Y}{Z^{2}}=\frac{\mathrm{d} \varnothing \sin \psi}{\sin ^{2} \sigma \sin \omega}
$$

and since $Z=v \sin \sigma \sin \omega$, we find these very fitting formulas:

$$
\begin{gathered}
X \mathrm{~d} Z-Z \mathrm{~d} X=v^{2} \mathrm{~d} \varnothing \sin \omega \cos \psi \text { and } \\
Y \mathrm{~d} Z-Z \mathrm{~d} Y=v^{2} \mathrm{~d} \varnothing \sin \omega \sin \psi .
\end{gathered}
$$

Next, we eliminate $\mathrm{d} Z$, firstly multiplying by $Y$, and then by $X$, and once the product is taken, it will give

$$
Z(X \mathrm{~d} Y-Y \mathrm{~d} X)=v^{2} \mathrm{~d} \varnothing \sin \omega(Y \cos \psi-X \sin \psi) .
$$

However, as we saw in $\S 7, Y \cos \psi-X \sin \psi=\frac{Z \cos \omega}{\sin \omega}$, this formula is reduced to a much simple expression

$$
X \mathrm{~d} Y-Y \mathrm{~d} X=v^{2} \mathrm{~d} \varnothing \cos \omega .
$$

\footnotetext{
${ }^{27}$ The application of the Law of Sines to the spherical triangle $N n Z$ does not give this expression. ${ }^{28}$ It is not known where this expression comes from.

${ }^{29}$ The claimed division does not lead to this expression.

${ }^{30}$ From the above observations, it appears that this result was forced by Euler. Nonetheless, this has no further consequences, since the same expression was obtained before by another method in $₫ 7$.

${ }^{31}$ This figure was incremented with more elements by the Translator.
} 
These formulas when combined with those then found at the beginning, namely

$$
X^{2}+Y^{2}+Z^{2}=v^{2} \text { and } \mathrm{d} X^{2}+\mathrm{d} Y^{2}+\mathrm{d} Z^{2}=\mathrm{d} v^{2}+v^{2} \mathrm{~d} \varnothing^{2}
$$

will be used with maximum advantage in the mechanical part, to the coordinates derived from the calculations, such that it provides to the next quantities of this sort, which use are retaken in Astronomy.

XI) However, those reductions of the Geometry clearly show that it makes no difference to which point $A$ and fixed plane $B A Y$ we wish to refer the motion of the point $Z$. In fact, if we envisage an astronomical use, it is of most interest, not only in which way the point $A$, as the center of motion is taken into account, but also that plane, to which the motion of the point $Z$ is referred to by longitude and latitude, because from this will depend the chief simplicity of the determination. To this end, it is necessary to consider how that choice should be made, together with the artifices, which so far have been devised, and only then, it can be used with some success, since the motion of the body, which is sought, should not disagree very much from the laws of Kepler, on account that the perturbations hand been very small. Moreover, when the motion is thus compared, such that the areas described around any point are nearly proportional to time, then this point is most suitable to be considered as that fixed point $A$. When that happens, if among the forces driving the body, one far exceeds the remaining, to that point this force should be directed to, then point $A$ will be suited to be accepted: therefore, if the question would be related to perturbations of a certain chief planet or of a comet, then point $A$ will be most suitable taken in the center of the Sun: if however, the perturbations in the motion of the Moon, or those made in another secondary planet should be defined; then it is proper be considered point $A$ in the center of the Earth or [in the center] of the primary planet, such that the force of the body declared in $Z$ impelling it to $A$, much exceeds the remaining forces to which this body is simultaneously driven.

XII) So, If the body $Z$ has been solicited by just one principal force, the body will be revolved regularly around point $A$ in a conical section, perpetually in the same plane, such that no matter in what way the fixed plane $B A Y$ is chosen, neither on how the nodal line nor the inclination and any mutation has been ever originated; however, meanwhile, the calculation, without doubt, has turned out very simple, if the fixed plane is chosen in the same plane of the motion. Truly, if the motion is disturbed by another celestial body, which motion is indeed also necessary to be assumed known in this investigation, the fixed plane can most conveniently be assumed as being congruent with the orbit of that disturbing body. Thus, if the perturbations of the Moon originated by the Sun are sought, the ecliptic plane, in which the Sun is seen to move from the Earth, as it the Earth] were the center of the motion $A$, will render the fixed plane $B A Y$, and no matter how the perturbation from another body is brought about, this plane, in which this body is seen to move from the center of motion $A$, should be selected. Yet, if this body itself is not moved in the same plane, then some medium plane can be most conveniently adopted; but the effort to adapt the calculation to this 
case is hardly considered, but, if its employment will become indeed necessary, it can be easily provided.

\section{MECHANICAL PART}

XIII) For the handling of the mechanical part, three bodies should be considered. The first, is the one which is putted in the center of the motion $A$, which exerts the main force in the body $Z$, which motion we investigate, such as it appears to an observer located in the very point $A$, let us then call the mass of the body positioned in this point $=A$.

The other body, by which action the motion of the body $Z$ is perturbed, that we assume is moving in any manner in the fixed plane $B A Y$ itself, such that its location can be assigned at any time. Be the mass of this body $=B$, and that now, in fact, it dwells in $S$, such that its distance to the central body $A S=u$, and the longitude or angle $B A S=\theta$, whence, from $S$, the perpendicular $S P$ is drawn to the fixed line $A B$, and be $A P=u \cos \theta$ and $P S=u \sin \theta$.

The third body is the one in $Z$ itself, which motion we are looking for, be its mass $=C$, and as before, we put its distance to the center of motion $A Z=v$, and calling the three orthogonal coordinates $A X=X, X Y=Y$ and $Y Z=Z$, which we obtain from the calculation by introducing the following elements:

1) longitude of the nodal line or angle $B A N=\psi$

2) Inclination of the orbit to the fixed plane $=\omega$

3) argument of latitude or angle $N A Z=\sigma$.

Finally, we consider that during the infinitesimal time $\mathrm{d} t$, the elementary angle $Z A Z=\mathrm{d} \varnothing$ is completed by the body $Z$. On the other hand, the relationships of these elements will be taken from the geometrical part.

XIV) Since the body $Z$ is driven to $A$ by a force $=\frac{A}{v^{2}}$, certainly, $A$ is attracted to $Z$ by a force $=\frac{C}{v^{2}}$, so that for the point $A$ could be considered at rest, the body $Z$ should be declared to be attracted to $A$ by a force $=\frac{A+C}{v^{2}},{ }^{32}$ which once

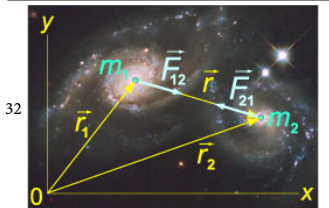

In a system of two bodies, the attraction force $F_{12}$ of the second body

acts on the first body of mass $m_{1}$. Similarly, the attraction force $F_{21}$ of the first body acts on the second body of mass $m_{2}$. Both forces $F_{12}$ and $F_{21}$ are equal and directed along $r$, where $r=r_{2}-r_{1}$. From Newton's second law, we can write the following differential equations describing the motion of each body: $m_{2} \frac{\mathrm{d}_{r_{2}}^{2}}{\mathrm{~d}^{2}}=-G \frac{m_{1} m_{2}}{r^{3}} r$ or $\frac{\mathrm{d}_{r_{1}}^{2}}{\mathrm{~d} t^{2}}=G \frac{m_{2}}{r^{3}} r, \frac{\mathrm{d}_{r_{2}}^{2}}{\mathrm{~d} t^{2}}=-G \frac{m_{1}}{r^{3}} r$, where $G$ is the gravitational constant. It follows from the last two equations that $\frac{\mathrm{d}_{r_{1}}^{2}}{\mathrm{~d} t^{2}}-\frac{\mathrm{d}_{r_{2}}^{2}}{\mathrm{~d} t^{2}}=G \frac{m_{2}}{r^{3}} r+G \frac{m_{1}}{r^{3}} r$, and then, $\frac{\mathrm{d}_{r}^{2}}{\mathrm{~d} t^{2}}=-G \frac{m_{1}+m_{2}}{r^{3}} r$. For $m_{1}=A, m_{2}=C, r=v$, and when the two bodies are collinear with $O$, results in $\frac{\mathrm{d}_{r}^{2}}{\mathrm{~d} t^{2}}=-G \frac{A+C}{v^{2}}$, where the minus sign means that these forces tend to shorten the distance between the two bodies. 
resolved according to the directions of the three coordinates give the following forces: according to $X A:=\frac{A+C}{v^{3}} \cdot X$; according to $Y X:=\frac{A+C}{v^{3}} \cdot Y$; according to $Z Y:=\frac{A+C}{v^{3}} \cdot Z$. Thereafter, for the force with which the body $Z$ is attracted towards $S$, we have: firstly, let us call, for simplicity, the distance $S Z=w$, such that the force $Z S$ is $=\frac{B}{w^{2}}$, which can be readily decomposed into the forces: according to $Z Y:=\frac{B}{w^{3}} Z$, and according to $Y S:=\frac{B}{w^{3}} Y S$, and from this, since $X P=u \cos \theta-X$ and $P S-X Y=u \sin \theta-Y$, we have the forces: according to $X P:=\frac{B}{w^{3}}(u \cos \theta-X)$ and according to $X Y:=\frac{B}{w^{3}}(u \sin \theta-Y) \cdot{ }^{33}$

Finally, because body $A$ is driven to $S$ by a force $=\frac{B}{u^{2}}$, the following components will be contrarily translated to $Z$ : according to $X A:=\frac{B}{u^{2}} \cos \theta$, and according to $Y X:=\frac{B}{u^{2}} \sin \theta$, which once collected with the other forces acting on the body $Z$ give:

1) Force according to $X A:=\frac{A+C}{v^{3}} X+\frac{B}{w^{3}}(X-u \cos \theta)+\frac{B}{u^{2}} \cos \theta$

2) Force according to $X Y:=\frac{A+C}{v^{3}} Y+\frac{B}{w^{3}}(Y-u \sin \theta)+\frac{B}{u^{2}} \sin \theta$

3) Force according to $Z Y:=\frac{A+C}{v^{3}} Z+\frac{B}{w^{3}} Z$,

which should be proportional to the acceleration of the body $Z$ in the same directions, and considering a constant element of time we have:

$$
\begin{gathered}
\mathrm{dd} X=-\propto \mathrm{d} t^{2}\left[\frac{A+C}{v^{3}} X+\frac{B}{w^{3}} X-B u \cos \theta\left(\frac{1}{w^{3}}-\frac{1}{u^{3}}\right)\right] \\
\mathrm{dd} Y=-\propto \mathrm{d} t^{2}\left[\frac{A+C}{v^{3}} Y+\frac{B}{w^{3}} Y-B u \sin \theta\left(\frac{1}{w^{3}}-\frac{1}{u^{3}}\right)\right] \\
\mathrm{dd} Z=-\propto \mathrm{d} t^{2}\left[\frac{A+C}{v^{3}} Z+\frac{B}{w^{3}} Z\right],
\end{gathered}
$$

where the constant $\propto$ depends on each particular type of motion, and can be defined from the apparent motion of the Sun.

$\mathrm{XV}$ ) However, before we consider these formulas further, we should precisely define the distance $S Z=w$, to introduce it afresh into the calculation. Since we have that:

$$
S Z^{2}=Z Y^{2}+X P^{2}+(P S-X Y)^{2} 34
$$

then,

\footnotetext{
${ }^{33}$ These are the components of the force according to $Y S$, projected in the directions $A X$ and $X Y$.

${ }^{34}$ The first term in the second hand-side of this expression was incorrectly written as $X Z^{2}$ in the original manuscript.
} 


$$
w^{2}=Z^{2}+X^{2}+Y^{2}+u^{2}-2 u X \cos \theta-2 u Y \sin \theta,
$$

which because $X^{2}+Y^{2}+Z^{2}=v^{2}$, can be reduced to:

$$
w^{2}=v^{2}+u^{2}-2 u(X \cos \theta+Y \sin \theta) .
$$

Moreover, introducing the expressions for $X$ an $Y$ found in $\$ 4$. above:

$$
X \cos \theta+Y \sin \theta=v[\cos \sigma \cos (\theta-\psi)+\sin \sigma \cos \omega \sin (\theta-\psi)],
$$

where the angle $\theta-\psi$ expresses the distance of the disturbing body $S$ to the nodal line or angle $N A S=\theta-\psi$, so that

$$
w^{2}=v^{2}+u^{2}-2 v u[\cos \sigma \cos (\theta-\psi)+\sin \sigma \cos \omega \sin (\theta-\psi)] .
$$

In fact, if now for brevity we call the angle $S A Z=\mu$, which denotes the distance of the body $Z$ to the disturbing body $S$ as seen from $A$, because $A Z=v$ and $A S=u$, it is also true that

$$
w^{2}=v^{2}+u^{2}-2 v u \cos \mu,
$$

whence, it will be concluded that:

$$
\cos \sigma \cos (\theta-\psi)+\sin \sigma \cos \omega \sin (\theta-\psi)=\cos \mu
$$

which can be easily proved by spherical trigonometry ${ }^{35}$. As seen in figure, since because $B N=\psi, N Z=\sigma$, and the angle $Y N Z=\omega$, if $B S=\theta$, then $N S=\theta-\psi$, and in the spherical triangle the side $S Z=\mu$ is determined in this very way from the sides $N Z=\sigma, N S=\theta-\psi$ with the intercepted angle $Z N S=\omega$.

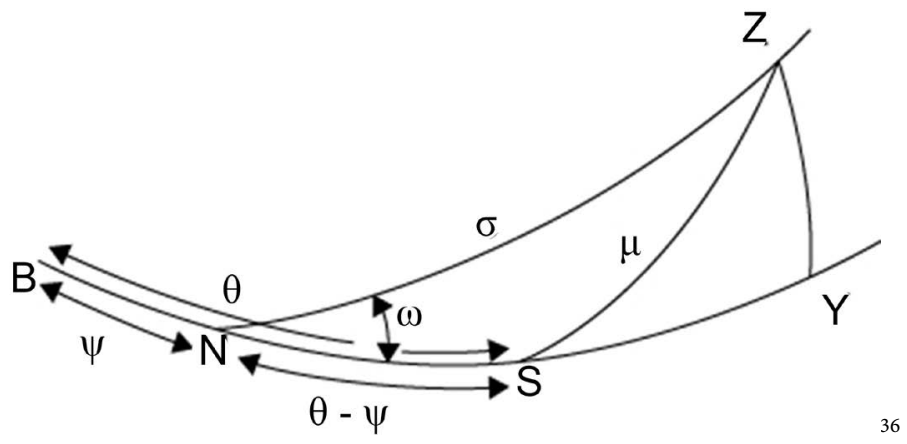

XVI) Although the three equations deducted from the principles of mechanics are enough for all determinations, because all the craft in them is certain, just as it enable us to derive them in a very suitable manner. Nonetheless, it is first required to offer at once the calculation of the multiplications: the first [equation] by $2 \mathrm{~d} X$, the second [equation] by $2 \mathrm{~d} Y$ and the third [equation] by $2 \mathrm{~d} Z$, and in only one [equation] they ought be gathered; since as we saw above

$$
\begin{gathered}
\mathrm{d} X^{2}+\mathrm{d} Y^{2}+\mathrm{d} Z^{2}=\mathrm{d} v^{2}+v^{2} \mathrm{~d} \varnothing^{2} \\
\text { and } X^{2}+Y^{2}+Z^{2}=v^{2}
\end{gathered}
$$

\footnotetext{
${ }^{35}$ This result comes from the application of the law of cosines to the spherical triangle of the figure. ${ }^{36}$ This figure was added by the Translator.
} 
then

$2 \mathrm{~d} X \mathrm{dd} X+2 \mathrm{~d} Y \mathrm{dd} Y+2 \mathrm{~d} Z \mathrm{dd} Z=\mathrm{d}\left(\mathrm{d} v^{2}+v^{2} \mathrm{~d} \varnothing^{2}\right)$ and $X \mathrm{~d} X+Y \mathrm{~d} Y+Z \mathrm{~d} Z=v \mathrm{~d} v$.

Hence, with the reminded calculation, the following equation will be obtained:

$$
\begin{aligned}
& \mathrm{d}\left(\mathrm{d} v^{2}+v^{2} \mathrm{~d} \varnothing^{2}\right) \\
& =-2 \propto \mathrm{d} t^{2}\left[\frac{A+C}{v^{2}} \mathrm{~d} v+\frac{B}{w^{3}} v \mathrm{~d} v-B u(\mathrm{~d} X \cos \theta+\mathrm{d} Y \sin \theta)\left(\frac{1}{w^{3}}-\frac{1}{u^{3}}\right)\right]
\end{aligned}
$$

where the formula $\mathrm{d} X \cos \theta+\mathrm{d} Y \sin \theta$ can be conveniently expanded. Truly, considering the formulas in $₫ 10$.a above, we have that

$$
\mathrm{d} X=\frac{X \mathrm{~d} Z}{Z}-\frac{v \mathrm{~d} \varnothing \cos \psi}{\sin \sigma} \text { and } \mathrm{d} Y=\frac{Y \mathrm{~d} Z}{Z}-\frac{v \mathrm{~d} \varnothing \sin \psi}{\sin \sigma}
$$

because $Z=v \sin \sigma \sin \omega$, whence

$$
\mathrm{d} X \cos \theta+\mathrm{d} Y \sin \theta=\frac{\mathrm{d} Z}{Z}(X \cos \theta+Y \sin \theta)-\frac{v \mathrm{~d} \varnothing \cos (\theta-\psi)}{\sin \sigma},
$$

however, we recently saw that

$$
X \cos \theta+Y \sin \theta=v[\cos \sigma \cos (\theta-\psi)+\sin \sigma \cos \omega \sin (\theta-\psi)]=v \cos \mu,
$$

and since, in fact,

$$
\begin{gathered}
\frac{\mathrm{d} Z}{Z}=\frac{\mathrm{d} v}{v}+\frac{\mathrm{d} \sigma \cos \sigma}{\sin \sigma}+\frac{\mathrm{d} \omega \cos \omega}{\sin \omega}, \text { or } \\
\frac{\mathrm{d} Z}{Z}=\frac{\mathrm{d} v}{v}+\frac{\mathrm{d} \sigma \cos \sigma}{\sin \sigma}+\frac{\mathrm{d} \psi \cos \omega}{\tan \sigma} \text { because } \mathrm{d} \omega=\frac{\mathrm{d} \psi \sin \omega}{\tan \sigma} .
\end{gathered}
$$

Since $\mathrm{d} \varnothing=\mathrm{d} \sigma+\mathrm{d} \psi \cos \omega$, then we get

$$
\frac{\mathrm{d} Z}{Z}=\frac{\mathrm{d} v}{v}+\frac{\mathrm{d} \varnothing}{\tan \sigma},
$$

therefore

$$
\begin{aligned}
& \mathrm{d} X \cos \theta+\mathrm{d} Y \sin \theta=\mathrm{d} v \cos \mu+\frac{v \mathrm{~d} \varnothing \cos \mu}{\tan \sigma}-\frac{v \mathrm{~d} \varnothing \cos (\theta-\psi)}{\sin \sigma} \\
& =\mathrm{d} v \cos \mu+\frac{v \mathrm{~d} \varnothing}{\sin \sigma}\left[\sin \sigma \cos \sigma \cos \omega \sin (\theta-\psi)+\cos ^{2} \sigma \cos (\theta-\psi)-\cos (\theta-\psi)\right]
\end{aligned}
$$

and thus,

$$
\mathrm{d} X \cos \theta+\mathrm{d} Y \sin \theta=\mathrm{d} v \cos \mu-v \mathrm{~d} \varnothing[\sin \sigma \cos (\theta-\psi)-\cos \sigma \cos \omega \sin (\theta-\psi)] .
$$

Therefore, the equation that we found transforms into:

$$
\begin{aligned}
& \mathrm{d}\left(\mathrm{d} v^{2}+v^{2} \mathrm{~d} \varnothing^{2}\right) \\
& =-2 \propto \mathrm{d} t^{2} \mathrm{~d} v\left[\frac{A+C}{v^{2}}+\frac{B v}{w^{3}}-B u \cos \mu\left(\frac{1}{w^{3}}-\frac{1}{u^{3}}\right)\right] \\
& -2 \propto \mathrm{d} t^{2} \mathrm{~d} \varnothing \cdot u v[\sin \sigma \cos (\theta-\psi)-\cos \sigma \cos \omega \sin (\theta-\psi)]\left(\frac{1}{w^{3}}-\frac{1}{u^{3}}\right)
\end{aligned}
$$

XVII) It is possible to deduce a more concise form for the expansion of the too complicated formula $\mathrm{d} X \cos \theta+\mathrm{d} Y \sin \theta$ from the proper values found for 
$X$ and $Y$. In fact these differentials are duly produced in case where the angles $\psi$ and $\omega$ are handled as constants and $\mathrm{d} \sigma$ is brought to $\mathrm{d} \varnothing$, then this differential give:

$$
\begin{aligned}
& \mathrm{d} X=\mathrm{d} v(\cos \sigma \cos \psi-\sin \sigma \cos \omega \sin \psi)-v \mathrm{~d} \varnothing(\sin \sigma \cos \psi+\cos \sigma \cos \omega \sin \psi) \\
& \mathrm{d} Y=\mathrm{d} v(\cos \sigma \sin \psi+\sin \sigma \cos \omega \cos \psi)-v \mathrm{~d} \varnothing(\sin \sigma \sin \psi-\cos \sigma \cos \omega \cos \psi)
\end{aligned}
$$

whence, it follows that

$$
\begin{aligned}
\mathrm{d} X \cos \theta+\mathrm{d} Y \sin \theta= & \mathrm{d} v[\cos \sigma \cos (\theta-\psi)+\sin \sigma \cos \omega \sin (\theta-\psi)] \\
& -v \mathrm{~d} \varnothing[\sin \sigma \cos (\theta-\psi)-\cos \sigma \cos \omega \sin (\theta-\psi)]
\end{aligned}
$$

to which I observe a more contracted form in figure where $N S=\theta-\psi$; $N Z=\sigma, S N Z=\omega$ and $S Z=\mu$, we will have, in the first place, as before: $\cos \sigma \cos (\theta-\psi)+\sin \sigma \cos \omega \sin (\theta-\psi)=\cos \mu$, then, in fact, if the angle $N Z S=\xi$ it is found that

$$
\cot \xi=\frac{\sin \sigma \cos (\theta-\psi)-\cos \sigma \cos \omega \sin (\theta-\psi)}{\sin \omega \sin (\theta-\psi)}{ }^{37}
$$

whence it is concluded that

$$
\begin{aligned}
& \sin \sigma \cos (\theta-\psi)-\cos \sigma \cos \omega \sin (\theta-\psi) \\
& =\frac{\sin \omega \sin (\theta-\psi) \cos \xi}{\sin \xi}=\sin \mu \cos \xi
\end{aligned}
$$

because: $\sin \xi: \sin (\theta-\psi)=\sin \omega: \sin \mu .^{38}$

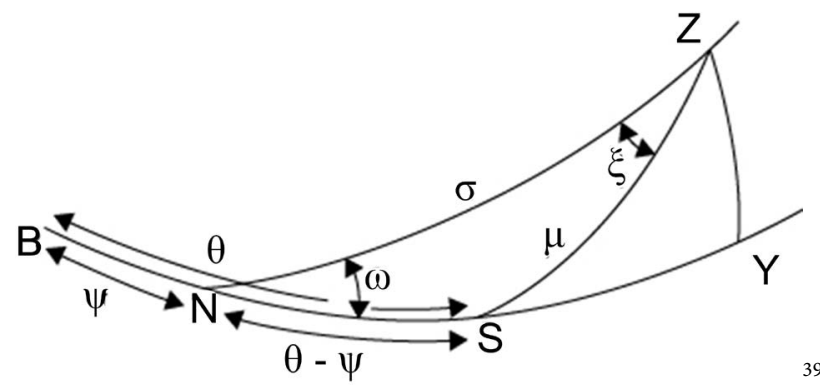

From these results we obtain

$$
\mathrm{d} X \cos \theta+\mathrm{d} Y \sin \theta=\mathrm{d} v \cos \mu-v \mathrm{~d} \varnothing \sin \mu \cos \xi .
$$

Or if in $Z$ we draw in the direction of the arch $N Z$ another normal arch, and on it, and from $S$, we draw the perpendicular to the spherical surface, which we call $=v$, then $\sin v=\sin \mu \cos \xi,{ }^{40}$ or

$$
\sin \sigma \cos (\theta-\psi)-\cos \sigma \cos \omega \sin (\theta-\psi)=\sin v
$$

and therefore, the equation containing the first determination will assume the ${ }^{37}$ This result comes from the application of the law of the tangent to the spherical triangle of the figure.

${ }^{38}$ This result comes from the application of the law of sines to the spherical triangle of the figure.

${ }^{39}$ This figure was added by the Translator.

${ }^{40}$ This geometrical construction is not clear. 
following form

$$
\begin{aligned}
& \mathrm{d}\left(\mathrm{d} v^{2}+v^{2} \mathrm{~d} \varnothing^{2}\right) \\
& =-2 \propto \mathrm{d} t^{2}\left[\frac{A+C}{v^{2}} \mathrm{~d} v+\frac{B v \mathrm{~d} v}{w^{3}}-B u(\mathrm{~d} v \cos \mu-v \mathrm{~d} \varnothing \sin v)\left(\frac{1}{w^{3}}-\frac{1}{u^{3}}\right)\right]
\end{aligned}
$$

XVIII) The two remaining determinations from the differential equations deducted from the principles of motion will be conveniently obtained by the following procedures: firstly, from the equations obtained in $\$ 14$, the subtraction of the first equation multiplied by $Y$ from the second equation multiplied by $X$ gives:

$$
X \mathrm{dd} Y-Y \mathrm{dd} X=-\propto \mathrm{d} t^{2} B u(Y \cos \theta-X \sin \theta)\left(\frac{1}{w^{3}}-\frac{1}{u^{3}}\right)
$$

or, when the values for $X$ and $Y$ are substituted into this expression results in

$$
\begin{aligned}
& X \mathrm{dd} Y-Y \mathrm{dd} X \\
& =\propto B v u \mathrm{~d} t^{2}[\cos \sigma \sin (\theta-\psi)-\sin \sigma \cos \omega \cos (\theta-\psi)]\left(\frac{1}{w^{3}}-\frac{1}{u^{3}}\right)
\end{aligned}
$$

Thus, since $X \mathrm{dd} Y-Y \mathrm{dd} X$ is the differential of $X \mathrm{~d} Y-Y \mathrm{~d} X$, then we have this equation

$$
\begin{aligned}
& \mathrm{d}\left(v^{2} \mathrm{~d} \varnothing \cos \omega\right) \\
& =\propto B v u \mathrm{~d} t^{2}[\cos \sigma \sin (\theta-\psi)-\sin \sigma \cos \omega \cos (\theta-\psi)]\left(\frac{1}{w^{3}}-\frac{1}{u^{3}}\right)
\end{aligned}
$$

In a similar way, from the first and third [equations of $\$ 14$ ] we deduce

$$
X \mathrm{dd} Z-Z \mathrm{dd} X=-\propto B \mathrm{BuZd} t^{2} \cos \theta\left(\frac{1}{w^{3}}-\frac{1}{u^{3}}\right)
$$

or

$$
X \mathrm{dd} Z-Z \mathrm{dd} X=-\propto B v u \mathrm{~d} t^{2} \cos \theta \sin \sigma \sin \omega\left(\frac{1}{w^{3}}-\frac{1}{u^{3}}\right)
$$

then giving

$$
\mathrm{d}\left(v^{2} \mathrm{~d} \varnothing \sin \omega \cos \psi\right)=-\propto B v u \mathrm{~d} t^{2} \cos \theta \sin \sigma \sin \omega\left(\frac{1}{w^{3}}-\frac{1}{u^{3}}\right) .
$$

Equally, from the second equation combined with the third equation results in

$$
Y \mathrm{dd} Z-Z \mathrm{dd} Y=-\propto B v u \mathrm{dt} t^{2} \sin \theta \sin \sigma \sin \omega\left(\frac{1}{w^{3}}-\frac{1}{u^{3}}\right)
$$

or

$$
\mathrm{d}\left(v^{2} \mathrm{~d} \varnothing \sin \omega \sin \psi\right)=-\propto B v u \mathrm{~d} t^{2} \sin \theta \sin \sigma \sin \omega\left(\frac{1}{w^{3}}-\frac{1}{u^{3}}\right)
$$

where it should be noted that only two determinations are contained in these three equations, and the third [equation] can be freely included with the two other [equations]. 
XIX) So, let us examine the two last [equations], and since their first members ought to be differentiated, and observing that the quantity $v^{2} \mathrm{~d} \varnothing \sin \omega$ is a unique quantity to both, then

$$
\begin{aligned}
& \cos \psi \mathrm{d}\left(v^{2} \mathrm{~d} \varnothing \sin \omega\right)-\mathrm{d} \psi \sin \psi v^{2} \mathrm{~d} \varnothing \sin \omega \\
& =-\propto B v u \mathrm{~d} t^{2} \cos \theta \sin \sigma \sin \omega\left(\frac{1}{w^{3}}-\frac{1}{u^{3}}\right) \\
& \sin \psi \mathrm{d}\left(v^{2} \mathrm{~d} \varnothing \sin \omega\right)+\mathrm{d} \psi \cos \psi v^{2} \mathrm{~d} \varnothing \sin \omega \\
& =-\propto B v u \mathrm{~d} t^{2} \sin \theta \sin \sigma \sin \omega\left(\frac{1}{w^{3}}-\frac{1}{u^{3}}\right)
\end{aligned}
$$

whence, eliminating $\mathrm{d}\left(v^{2} \mathrm{~d} \varnothing \sin \omega\right)$ results in

$$
\mathrm{d} \psi v^{2} \mathrm{~d} \varnothing \sin \omega=-\propto B v u \mathrm{~d} t^{2} \sin \sigma \sin \omega \sin (\theta-\psi)\left(\frac{1}{w^{3}}-\frac{1}{u^{3}}\right)
$$

and thus the variation of the nodal line is defined such that

$$
\mathrm{d} \psi=\frac{-\propto B u \mathrm{~d} t^{2} \sin \sigma \sin (\theta-\psi)}{v \mathrm{~d} \varnothing}\left(\frac{1}{w^{3}}-\frac{1}{u^{3}}\right)
$$

from which, at the same time, the variation of the inclination is obtained from $\frac{\mathrm{d} \omega}{\sin \omega}=\frac{\mathrm{d} \psi}{\tan \sigma}$, and, on the other hand, once the member $v^{2} \mathrm{~d} \varnothing \sin \omega$ is eliminated from both original equations, the following equation is obtained

$$
\mathrm{d}\left(v^{2} \mathrm{~d} \varnothing \sin \omega\right)=-\propto B v u \mathrm{~d} t^{2} \sin \sigma \sin \omega \cos (\theta-\psi)\left(\frac{1}{w^{3}}-\frac{1}{u^{3}}\right) .
$$

Writing the first equation in the following form:

$$
\begin{aligned}
& \cos \omega \mathrm{d}\left(v^{2} \mathrm{~d} \varnothing\right)-\mathrm{d} \omega \sin \omega v^{2} \mathrm{~d} \varnothing \\
& =\propto B v u \mathrm{~d} t^{2}[\cos \sigma \sin (\theta-\psi)-\sin \sigma \cos \omega \cos (\theta-\psi)]\left(\frac{1}{w^{3}}-\frac{1}{u^{3}}\right)
\end{aligned}
$$

and expanding the first member of the last equation, gives

$$
\begin{aligned}
& \sin \omega \mathrm{d}\left(v^{2} \mathrm{~d} \varnothing\right)+\mathrm{d} \omega \cos \omega v^{2} \mathrm{~d} \varnothing \\
& =-\propto B v u \mathrm{~d} t^{2}[\sin \sigma \sin \omega \cos (\theta-\psi)]\left(\frac{1}{w^{3}}-\frac{1}{u^{3}}\right)
\end{aligned}
$$

Eliminating $\mathrm{d} \omega$ between the last two equations, gives

$$
\mathrm{d}\left(v^{2} \mathrm{~d} \varnothing\right)=-\propto B v u \mathrm{~d} t^{2} \mathrm{~d} t^{2}[\sin \sigma \cos (\theta-\psi)-\cos \sigma \cos \omega \sin (\theta-\psi)]\left(\frac{1}{w^{3}}-\frac{1}{u^{3}}\right)
$$

or

$$
\mathrm{d}\left(v^{2} \mathrm{~d} \varnothing\right)=-\propto B v u \mathrm{~d} t^{2} \sin v\left(\frac{1}{w^{3}}-\frac{1}{u^{3}}\right)
$$

which is the other determination required to be sought.

$\mathrm{XX}$ ) Let us multiply this last equation by $2 v^{2} \mathrm{~d} \varnothing$, and leaving one integral just indicated, we will have the following expression 


$$
v^{4} \mathrm{~d} \varnothing^{2}=-2 \propto B \mathrm{~d} t^{2} \int v^{3} u \mathrm{~d} \varnothing u \sin v\left(\frac{1}{w^{3}}-\frac{1}{u^{3}}\right){ }^{41}
$$

this equation contains the relation between the elementary angle $\mathrm{d} \varnothing$ and the infinitesimal time $\mathrm{d} t$, where, in fact, it is clear that if the mass of de disturbing body $B$ would fade away, then $v^{2} \mathrm{~d} \varnothing$ would be proportional to the time $\mathrm{d} t$, or the areas described around $A$ are proportional to time. If to this equation, it is first added the one found in $₫ 17$, and equally integrated to the extent possible, then

$$
\begin{aligned}
\mathrm{d} v^{2}+v^{2} \mathrm{~d} \varnothing^{2}= & 2 \propto \mathrm{d} t^{2}(A+C)\left(\frac{1}{v}-\frac{1}{f}\right)-2 \propto B \mathrm{~d} t^{2} \int \frac{v \mathrm{~d} v}{w^{3}} \\
& +2 \propto B \mathrm{~d} t^{2} \int u(\mathrm{~d} v \cos \mu-v \mathrm{~d} \varnothing \sin v)\left(\frac{1}{w^{3}}-\frac{1}{u^{3}}\right)
\end{aligned}
$$

the above equation compares the variation of the distance $v$ with the element $\mathrm{d} \varnothing$ or with the infinitesimal time $\mathrm{d} t$, which are two particular characteristics to look for the motion of the body $Z$ in its own orbit. Besides, we have, in fact, for the variation of orbit itself the following:

$$
\begin{gathered}
\mathrm{d} \psi=\frac{-\propto B u \mathrm{~d} t^{2} \sin \sigma \sin (\theta-\psi)}{v \mathrm{~d} \varnothing}\left(\frac{1}{w^{3}}-\frac{1}{u^{3}}\right) \\
\frac{\mathrm{d} \omega}{\sin \omega}=\frac{-\propto B u \mathrm{~d} t^{2} \cos \sigma \sin (\theta-\psi)}{v \mathrm{~d} \varnothing}\left(\frac{1}{w^{3}}-\frac{1}{u^{3}}\right)=\frac{\mathrm{d} \psi}{\tan \sigma}
\end{gathered}
$$

And finally, it can be recalled the relation between the argument of the latitude $\sigma$ to these same elements, given by $\mathrm{d} \sigma=\mathrm{d} \varnothing-\mathrm{d} \psi \cos \omega$.

XXI) The element of time $\mathrm{d} t$ with the constant $\propto$ will be taken away from the calculation in a most convenient way, if a certain motion that is regular and known is introduced, like the mean motion of the Sun, or of another body, which is revolved around the center of the forces in a uniform circle. Then, let us put around the body located in $A$, which mass is $=\mathfrak{A}$, the other body, which mass $=\mathfrak{C}$, at a distance $=a$, in a circle, so that it circulates in a time $t$ an angle $\tau$ proportional to it, and our equations can be adapted to this case, once these are established: $A=\mathfrak{A}, C=\mathfrak{C}$ and $B=0$, and then $v=a$ and $\mathrm{d} \varnothing=\mathrm{d} \tau$. Then, the motion that we assume to be known, is controlled by these two equations

$$
v^{4} \mathrm{~d} \varnothing^{2}=2 \propto D \mathrm{~d} t^{2} \text { and } \mathrm{d} v^{2}+v^{2} \mathrm{~d} \varnothing^{2}=2 \propto \mathrm{d} t^{2}(\mathfrak{A}+\mathfrak{C})\left(\frac{1}{v}-\frac{1}{f}\right)
$$

where the first constants $D$ and $f$ can be conveniently defined for this case. To this end, from the first $2 \propto \mathrm{d} t^{2}=\frac{v^{4} \mathrm{~d} \varnothing^{2}}{D}$ which, when substituted into the second, gives $\mathrm{d} v^{2}+v^{2} \mathrm{~d} \varnothing^{2}=\frac{(\mathfrak{A}+\mathfrak{C}) v^{4} \mathrm{~d} \varnothing^{2}}{D}\left(\frac{1}{v}-\frac{1}{f}\right)$ or

$D f \mathrm{~d} v^{2}+D f v^{2} \mathrm{~d} \varnothing^{2}=(\mathfrak{A}+\mathfrak{C}) v^{3} \mathrm{~d} \varnothing^{2}(f-v)$, from which

${ }^{41}$ The number two in bold should not be there. 


$$
\mathrm{d} \varnothing=\frac{\mathrm{d} v \sqrt{D f}}{v \sqrt{(\mathfrak{A}+\mathfrak{C}) v(f-v)-D f}},
$$

a certain constant value of $v$ itself should be satisfactorily attributed to this differential equation, for which the denominator fades away, however, as I have exposed in another place, this approach is not possible to be admitted to the integral, unless the factor of denomination ${ }^{42}$ fades away to be of a minimum dimension of one, whence it is necessary that under the radical sign the same factor appears in a pair or squared, such that it reduces to the same value, so that the differential of the quantity placed after the sign reproduces the same factor. Therefore, let us place that differential $=0$, and $v=\frac{1}{2} f$, which under the hypothesis that $v=a$, then $f=2 a$, and in this case the denominator itself is then equal to $(\mathfrak{A}+\mathfrak{C}) a^{2}-2 D a$, which, when equated to zero, gives $D=\frac{1}{2}(\mathfrak{A}+\mathfrak{C}) a$ Now, in the other equation be considered that $v=a$ and $\mathrm{d} \varnothing=\mathrm{d} \tau$, then $a^{4} \mathrm{~d} \tau^{2}=\propto(\mathfrak{A}+\mathfrak{C}) a \mathrm{~d} t^{2}$ or $\propto \mathrm{d} t^{2}=\frac{a^{3} \mathrm{~d} \tau^{2}}{\mathfrak{A}+\mathfrak{C}}$.

XXII) Then, since the knowledge of the motion at any given time $t$ allows the determination of the mean motion $\tau$, here the time variable in our calculation will be redefined, by writing in the place of $\propto \mathrm{d} t^{2}$ the value just found of $\frac{a^{3} \mathrm{~d} \tau^{2}}{\mathfrak{A}+\mathfrak{C}}$. Next, to render our formulas simpler, let us put $\frac{A+C}{\mathfrak{A}+\mathfrak{C}}=m$ and $B=n(A+C)$, and then $\propto \mathrm{dt} t^{2}(A+C)=m a^{3} \mathrm{~d} \tau^{2}$ and $\propto B \mathrm{dt} t^{2}=m n a^{3} \mathrm{~d} \tau^{2}$ where it should be noted that the perturbations will be minimum if the terms affected by the number $n$ are minimum. Then our equations will assume the following forms:

1) $v^{4} \mathrm{~d} \varnothing^{2}=-2 m n a^{3} \mathrm{~d} \tau^{2} \int v^{3} u \mathrm{~d} \varnothing \sin v\left(\frac{1}{w^{3}}-\frac{1}{u^{3}}\right)$

2)

$$
\begin{aligned}
\mathrm{d} v^{2}+v^{2} \mathrm{~d} \varnothing^{2}= & 2 m a^{3} \mathrm{~d} \tau^{2}\left(\frac{1}{v}-\frac{1}{f}\right)-2 m n a^{3} \mathrm{~d} \tau^{2} \int \frac{v \mathrm{~d} v}{w^{3}} \\
& +2 m n a^{3} \mathrm{~d} \tau^{2} \int u(\mathrm{~d} v \cos \mu-v \mathrm{~d} \varnothing \sin v)\left(\frac{1}{w^{3}}-\frac{1}{u^{3}}\right)
\end{aligned}
$$

3) $\mathrm{d} \psi=-m n a^{3} \mathrm{~d} \tau^{2} \frac{u \sin \sigma \sin (\theta-\psi)}{v \mathrm{~d} \varnothing}\left(\frac{1}{w^{3}}-\frac{1}{u^{3}}\right)$

4) $\frac{\mathrm{d} \omega}{\sin \omega}=-m n a^{3} \mathrm{~d} \tau^{2} \frac{u \cos \sigma \sin (\theta-\psi)}{v \mathrm{~d} \varnothing}\left(\frac{1}{w^{3}}-\frac{1}{u^{3}}\right)=\frac{\mathrm{d} \psi}{\tan \sigma}$

5) $\mathrm{d} \sigma=\mathrm{d} \varnothing+m n a^{3} \mathrm{~d} \tau^{2} \cos \omega \frac{u \sin \sigma \sin (\theta-\psi)}{v \mathrm{~d} \varnothing}\left(\frac{1}{w^{3}}-\frac{1}{u^{3}}\right)=\mathrm{d} \varnothing-\mathrm{d} \psi \cos \omega$

with these equations, the whole motion of the body $Z$ with all the perturbations originated by the action of the body $S$ can be determined: where, especially regarded to the integral formulas, which in the first two equations are affected only by the perturbations, being sufficient that these values are selected as close to the real ones, from which the task of approximations to these integrals can hardly be ${ }^{42}$ It is simply the result of the division of the ratio. 
considered an impediment. Nonetheless, I will expose soon the method to such an extent as to liberate the calculation of these integrals.

XXIII) Meanwhile, for the sake of brevity, let us consider that:

$$
\begin{gathered}
\int v^{3} u \mathrm{~d} \varnothing u \sin v\left(\frac{1}{w^{3}}-\frac{1}{u^{3}}\right)=P \\
\int \frac{v \mathrm{~d} v}{w^{3}}=Q \\
\int u(\mathrm{~d} v \cos \mu-v \mathrm{~d} \varnothing \sin v)\left(\frac{1}{w^{3}}-\frac{1}{u^{3}}\right)=R
\end{gathered}
$$

then, the first two previous equations are contracted to these forms:

1) $v^{4} \mathrm{~d} \varnothing^{2}=2 m a^{3} \mathrm{~d} \tau^{2}(D-n P)$

2) $\mathrm{d} v^{2}+v^{2} \mathrm{~d} \varnothing^{2}=2 m a^{3} \mathrm{~d} \tau^{2}\left[\frac{1}{v}-\frac{1}{f}-n(Q-R)\right]$

which alone accomplishes all the task, if the body $Z$ is moved in the same plane, in which we assume that the disturbing body $S$ is circulating, the remaining equations for the motion, that is declared to pertain to the latitude, the solution of these suffers much less difficulties, since all the efforts should be consumed in the two previous [equations]. Thenceforth, once the element $\mathrm{d} \tau$ has been eliminated, arises this equation

$$
(D-n P)\left(\mathrm{d} v^{2}+v^{2} \mathrm{~d} \varnothing^{2}\right)=v^{4} \mathrm{~d} \varnothing^{2}\left[\left(\frac{1}{v}-\frac{1}{f}\right)-n(Q-R)\right]
$$

whence the following equation is obtained

$$
\mathrm{d} \varnothing=\frac{\mathrm{d} v \sqrt{(D-n P)}}{v \sqrt{\left(v-\frac{v^{2}}{f}-n v^{2}(Q-R)-D+n P\right)}}
$$

and furthermore

$$
2 m a^{3} \mathrm{~d} \tau^{2}=\frac{v^{2} \mathrm{~d} v^{2}}{v-\frac{v^{2}}{f}-n v^{2}(Q-R)-D+n P}
$$

or

$$
a \mathrm{~d} \tau \sqrt{2 m a}=\frac{v \mathrm{~d} v}{\sqrt{-D+n P+v-v^{2}\left(\frac{1}{f}+n Q-n R\right)}}
$$

the integration of these formulas would be available in case the fraction $n$ or the perturbations disappeared.

XXIV) We can represent that equation in this form:

$$
\frac{\mathrm{d} v}{v^{2}} \sqrt{(D-n P)}=\mathrm{d} \varnothing \sqrt{\left(-\frac{1}{f}-n(Q-R)+\frac{1}{v}-\frac{D-n P}{v^{2}}\right)}
$$

and since we know that $A Z=v$, then, it would become maximum or minimum 
when the quantity under the radical sign vanishes. However, not only in Astronomy that these places are of primary importance, wherever the body $Z$ is said to move along a segment of an arch, but even so, the choice of this important fact is at our disposal, with which the disturbed motion could be very neatly compared with the regular motion, and thus be capable to assign the aberrations from it. However, in a convenient way, this will be provided by introducing into the calculation a new angle $\Upsilon$, which in astronomy is called the true anomaly ${ }^{43}$, and it is chosen in such way that either by reducing or increasing the [angular] distance between two lines according to the maximum or minimum value of $v$. Therefore, with the purpose of approximating the real motion to a regular motion made along an ellipse, we now define $v=\frac{p}{1+q \cos \Upsilon}$, so that now the motion conforms to the regular motion along such an ellipse, in which the semi-latus rectum is $=p$, the eccentricity $=q$, and thus the semi-major axis $=\frac{p}{1-q^{2}}$, and the true anomaly arising from the [major] axis $=\Upsilon$. Then, it can be easily perceived that because of the perturbations, the aspect of this ellipse changes continuously, whence not only the anomaly $\Upsilon$ but also the letters $p$ and $q$ are expected to vary, and these variations are now investigated.

$\mathrm{XXV}$ ) For that investigation to be rendered easier, let us introduce, for the sake of brevity, the following:

$$
\frac{1}{f}-n(Q-R)=M \text { and } D-n P=N
$$

such that we have this evolved form

$$
\frac{\mathrm{d} v}{v^{2}} \sqrt{(D-n P)}=\mathrm{d} \varnothing \sqrt{\left(-M+\frac{1}{v}-\frac{N}{v^{2}}\right)} .
$$

Now that we have $v=\frac{p}{1+q \cos \Upsilon}$ or $\frac{1}{v}=\frac{1+q \cos \Upsilon}{p}$, by hypothesis, not only for the case where $\Upsilon=0$, which gives $\frac{1}{v}=\frac{1+q}{p}$, as well as for the case where $\Upsilon=180^{\circ}$, which gives $\frac{1}{v}=\frac{1-q}{p}$, the quantity $-M+\frac{1}{v}-\frac{N}{v^{2}}$ should go off to zero $^{44}$, and then, from these two situations arise the equations:

$$
-M+\frac{1+q}{p}-\frac{N(1+q)^{2}}{p^{2}}=0
$$

and

\footnotetext{
${ }^{43}$ The true anomaly $(Y)$ represents the real geometric angle in the plane of the elliptic, between periapsis (closest approach to the central body) and the position of the orbiting object at any given time. Argument of periapsis ( $\beta$ ) defines the orientation of an elliptical orbit in the orbital plane, as an angle measured from the ascending node to the periapsis (the closest point the celestial body [e.g Moon] comes to the central body [e.g Earth] around which it orbits).

${ }^{44} \mathrm{By}$ equating this quantity to zero, Euler is somehow searching for the maximum and minimum values of $v$, which translates into finding the major and minor axes of the ellipse.
} 


$$
-M+\frac{1-q}{p}-\frac{N(1-q)^{2}}{p^{2}}=0
$$

and by subtraction they give $\frac{2 q}{p}-\frac{4 N q}{p^{2}}=0$, such that $p=2 N$, or $N=\frac{1}{2} p$, whence $M=\frac{1+q}{p}-\frac{(1+q)^{2}}{2 p}=\frac{1-q^{2}}{2 p}$.

But if we put the semi-major axis of our ellipse $=r$ such that $r=\frac{p}{1-q^{2}}$, then $M=\frac{1}{2 r}$, and thus

$$
\frac{1}{f}-n(Q-R)=\frac{1}{2 r} \text { and } D-n P=\frac{1}{2} p .
$$

XXVI) When these values are substituted in our equation results in:

$$
\frac{\mathrm{d} v}{v^{2}} \sqrt{\frac{1}{2} p}=\mathrm{d} \varnothing \sqrt{\left(\frac{-1+q^{2}}{2 p}+\frac{1}{v}-\frac{p}{2 v^{2}}\right)}
$$

and when we write for $\frac{1}{v}$ in the right-hand side of this equation, the value $\frac{1+q \cos \Upsilon}{p}$, then we have that:

$$
\left.\frac{\mathrm{d} v}{v^{2}} \sqrt{\frac{1}{2} p}=\mathrm{d} \varnothing \sqrt{\left(\frac{-1+q^{2}}{2 p}+\frac{1+q \cos \Upsilon}{p}-\frac{1+2 q \cos \Upsilon+q^{2} \cos ^{2} \Upsilon}{2 p}\right)}\right)^{45}
$$

and thus,

$$
\frac{\mathrm{d} v}{v^{2}} \sqrt{\frac{1}{2} p}=\mathrm{d} \varnothing \sqrt{\frac{q^{2}-q^{2} \cos ^{2} \Upsilon}{2 p}}=\frac{q \mathrm{~d} \varnothing \sin \Upsilon}{\sqrt{2 p}}
$$

So, we have that $\frac{\mathrm{d} v}{v^{2}}=\frac{q \mathrm{~d} \varnothing}{p} \sin \Upsilon$; whence, surely, as we have anticipated, we certainly recognize that as the anomaly fades away with the sine of $\Upsilon$, at the same time, the differential of $v$ goes off to zero, and, therefore, it passes over a maximum or a minimum value. Hence, indeed, the increment of the distance $v$, in general, is reduced to the element $\mathrm{d} \varnothing$, which itself can now be compared with the known element $\mathrm{d} \tau$, and because $D-n P=\frac{1}{2} p$, we have that

$$
v^{4} \mathrm{~d} \varnothing^{2}=m a^{3} p \mathrm{~d} \tau^{2} \quad \text { or } v^{2} \mathrm{~d} \varnothing=a \mathrm{~d} \tau \sqrt{m a p}
$$

And since $\frac{1}{v}=\frac{1+q \cos \Upsilon}{p}$, then

$$
\frac{\mathrm{d} v}{v^{2}}=\frac{\mathrm{d} p(1+q \cos \Upsilon)}{p^{2}}-\frac{\mathrm{d} q \cos \Upsilon+q \mathrm{~d} \Upsilon \sin \Upsilon}{p}
$$

which turned out equal to the expression $\frac{q \mathrm{~d} \varnothing}{p} \sin \Upsilon$, resulting in

${ }^{45}$ There are errors in the signs of the last term under the radical which were corrected. 


$$
q(\mathrm{~d} \varnothing-\mathrm{d} \Upsilon) \sin \Upsilon=\frac{\mathrm{d} p}{p}(1+q \cos \Upsilon)-\mathrm{d} q \cos \Upsilon=\frac{\mathrm{d} p}{v}-\mathrm{d} q \cos \Upsilon,
$$

which involves new relations among differentials.

XXVII) The remaining determinations must be sought from the formulas found above:

$$
p=2 D-2 n P \text { and } \frac{1}{r}=\frac{1-q^{2}}{p}=\frac{2}{f}-2 n(R-Q),
$$

which once differentiated, and substituting the restituted values shown above for $P, Q, R$, give

$$
\begin{gathered}
\mathrm{d} p=-2 n \mathrm{~d} P=-2 n v^{3} u \mathrm{~d} \varnothing \sin v\left(\frac{1}{w^{3}}-\frac{1}{u^{3}}\right) \\
\mathrm{d}\left(\frac{1}{r}\right)=\mathrm{d}\left(\frac{1-q^{2}}{p}\right)=2 n \mathrm{~d} Q-2 n \mathrm{~d} R \\
=\frac{2 n v \mathrm{~d} v}{w^{3}}-2 n u(\mathrm{~d} v \cos \mu-v \mathrm{~d} \varnothing \sin v)\left(\frac{1}{w^{3}}-\frac{1}{u^{3}}\right)
\end{gathered}
$$

However, since $\mathrm{d} v=\frac{q v^{2} \mathrm{~d} \varnothing \sin \Upsilon}{p}=\frac{q v \mathrm{~d} \varnothing \sin \Upsilon}{1+q \cos \Upsilon}$, then, the last differential reduced to the element $\mathrm{d} \varnothing$ transforms into

$$
\begin{aligned}
\mathrm{d}\left(\frac{1}{r}\right) & =\mathrm{d}\left(\frac{1-q^{2}}{p}\right) \\
& =\frac{2 n q v^{3} \mathrm{~d} \varnothing \sin \Upsilon}{p w^{3}}-2 n v u \mathrm{~d} \varnothing\left(\frac{q \cos \mu \sin \Upsilon}{1+q \cos \Upsilon}-\sin v\right)\left(\frac{1}{w^{3}}-\frac{1}{u^{3}}\right)
\end{aligned}
$$

It is true that $\mathrm{d}\left(\frac{1-q^{2}}{p}\right)=\frac{-\mathrm{d} p}{p^{2}}\left(1-q^{2}\right)-\frac{2 q \mathrm{~d} q}{p}$, and then

$$
q \mathrm{~d} q=-\frac{\mathrm{d} p}{2 p}\left(1-q^{2}\right)-\frac{p}{2} \mathrm{~d}\left(\frac{1-q^{2}}{p}\right),
$$

from which it is conclude that

$$
\begin{aligned}
q \mathrm{~d} q= & +\frac{n\left(1-q^{2}\right)}{p} v^{3} u \mathrm{~d} \varnothing \sin v\left(\frac{1}{w^{3}}-\frac{1}{u^{3}}\right)-\frac{n q v^{3} \mathrm{~d} \varnothing \sin \Upsilon}{w^{3}} \\
& +n p v u \mathrm{~d} \varnothing\left(\frac{q \cos \mu \sin \Upsilon}{1+q \cos \Upsilon}-\sin v\right)\left(\frac{1}{w^{3}}-\frac{1}{u^{3}}\right)
\end{aligned}
$$

which, since $v=\frac{p}{1+q \cos \Upsilon}$, it is contracted into the following form

$$
\begin{aligned}
q \mathrm{~d} q= & n v^{2} u \mathrm{~d} \varnothing\left(\frac{1}{w^{3}}-\frac{1}{u^{3}}\right)\left[q \cos \mu \sin \Upsilon-\frac{q \sin v\left(q+2 \cos \Upsilon+q \cos ^{2} \Upsilon\right)}{1+q \cos \Upsilon}\right] \\
& -\frac{n q v^{3} \mathrm{~d} \varnothing \sin \Upsilon}{w^{3}}
\end{aligned}
$$

which, once divided by $q$, gives 


$$
\begin{aligned}
\mathrm{d} q= & n v^{2} u \mathrm{~d} \varnothing\left(\frac{1}{w^{3}}-\frac{1}{u^{3}}\right)\left[\cos \mu \sin \Upsilon-\frac{\sin v\left(q+2 \cos \Upsilon+q \cos ^{2} \Upsilon\right)}{1+q \cos \Upsilon}\right] \\
& -\frac{n v^{3} \mathrm{~d} \varnothing \sin \Upsilon}{w^{3}}
\end{aligned}
$$

Finally, when this expression is substituted into the formula $q(\mathrm{~d} \varnothing-\mathrm{d} \Upsilon) \sin \Upsilon=\frac{\mathrm{d} p}{v}-\mathrm{d} q \cos \Upsilon$, the resulting expression, once divided by $\sin \Upsilon$, is

$$
\begin{aligned}
q(\mathrm{~d} \varnothing-\mathrm{d} \Upsilon)= & \frac{n v^{3} \mathrm{~d} \varnothing \cos \Upsilon}{w^{3}}-n v^{2} u \mathrm{~d} \varnothing\left(\frac{1}{w^{3}}-\frac{1}{u^{3}}\right) \\
& \times\left[\cos \mu \cos \Upsilon+\frac{\sin v \sin \Upsilon(2+q \cos \Upsilon)}{1+q \cos \Upsilon}\right]
\end{aligned}
$$

XXVII) Therefore, now we have all the quantities that enter into our calculation, and we have revealed the sudden increment in the element $\mathrm{d} \varnothing$, which in the same infinitesimal time $\mathrm{d} t$ is completed by the here introduced angle $\mathrm{d} \tau$ according to the mean motion, whence, we can easily assign that increment for any minimum time. Hence, firstly, the relation between the elementary angle $\mathrm{d} \varnothing$ and $\mathrm{d} \tau$ is expressed by the following formula:

$$
v^{2} \mathrm{~d} \varnothing=a \mathrm{~d} \tau \sqrt{m a p} \text { whence, we have that } m a^{3} \mathrm{~d} \tau^{2}=\frac{1}{p} v^{4} \mathrm{~d} \varnothing^{2}
$$

next, if we consider that $v=\frac{p}{1+q \cos \Upsilon}$, and that $r=\frac{p}{1-q^{2}}$, then we will have that:

1) $\mathrm{d} p=-2 n v^{3} u \mathrm{~d} \varnothing \sin v\left(\frac{1}{w^{3}}-\frac{1}{u^{3}}\right)$

2)

$$
\mathrm{d}\left(\frac{1}{r}\right)=\frac{2 n q v^{3} \mathrm{~d} \varnothing \sin \Upsilon}{p w^{3}}-\frac{2 n v^{2} u \mathrm{~d} \varnothing}{p}
$$

$$
\times[q \cos \mu \sin \Upsilon-(1+q \cos \Upsilon) \sin v]\left(\frac{1}{w^{3}}-\frac{1}{u^{3}}\right)
$$

3)

$$
\begin{aligned}
\mathrm{d} q= & n v^{2} u \mathrm{~d} \varnothing\left(\frac{1}{w^{3}}-\frac{1}{u^{3}}\right)\left[\cos \mu \sin \Upsilon-\frac{\left(q+2 \cos \Upsilon+q \cos ^{2} \Upsilon\right) \sin v}{1+q \cos \Upsilon}\right] \\
& -\frac{n v^{3} \mathrm{~d} \varnothing \sin \Upsilon}{w^{3}} \\
\mathrm{~d} \Upsilon= & \mathrm{d} \varnothing-\frac{n v^{3} \mathrm{~d} \varnothing \cos \Upsilon}{q w^{3}}-\frac{n v^{2} u \mathrm{~d} \varnothing}{q}\left(\frac{1}{w^{3}}-\frac{1}{u^{3}}\right)
\end{aligned}
$$

4)

$$
\times\left[\cos \mu \cos \Upsilon+\frac{(2+q \cos \Upsilon) \sin v \sin \Upsilon}{1+q \cos \Upsilon}\right]
$$

knowing that

$$
\cos \mu=\cos \sigma \cos (\theta-\psi)+\sin \sigma \cos \omega \sin (\theta-\psi)
$$

and that 


$$
\sin v=\sin \sigma \cos (\theta-\psi)-\cos \sigma \cos \omega \sin (\theta-\psi)
$$

where it should be noted that $\mathrm{d} \varnothing-\mathrm{d} \Upsilon$ designates the increment of the arc described by the celestial body along the orbit itself.

Third, for the motion in the latitude we have these formulas:

1) $\mathrm{d} \psi=-\frac{n v^{3} u \mathrm{~d} \varnothing \sin \sigma \sin (\theta-\psi)}{p}\left(\frac{1}{w^{3}}-\frac{1}{u^{3}}\right)$

2) $\frac{\mathrm{d} \omega}{\sin \omega}=-\frac{n v^{3} u \mathrm{~d} \varnothing \cos \sigma \sin (\theta-\psi)}{p}\left(\frac{1}{w^{3}}-\frac{1}{u^{3}}\right)=\frac{\mathrm{d} \psi}{\tan \sigma}$

3) $\mathrm{d} \sigma=\mathrm{d} \varnothing+\frac{n v^{3} u \mathrm{~d} \varnothing \cos \omega \sin \sigma \sin (\theta-\psi)}{p}\left(\frac{1}{w^{3}}-\frac{1}{u^{3}}\right)$

or $\mathrm{d} \sigma=\mathrm{d} \varnothing-\mathrm{d} \psi \cos \omega$.

XXIX) Nothing more would be desired, if I could perform the integration of these equations, since then, any kind of perturbation could be defined, no matter great it would have been. But, since the forces of the Analysis have not yet increased to such an extent, it is fit to appeal to approximations, from which, hopefully, we could expect some success, in case the perturbations would be considered as being small: because then, the values of the quantities $p$ and $q$ would be changed by small amounts, having been affected by the letter $n$ in the integration of the formulas, such that, without error, can be considered just as constants, since, indeed, later on, the necessary corrections, obtained by the usual methods, can be applied without difficulties. However, in case we consider that the eccentricity is rather big, we will have difficulties, which, however, in order to be overcome, it will be possible to apply certain artifices, in which, indeed, the best way to succeed, is to consider that the eccentricity $q$, varies little from unity, such as in an almost parabolic orbit as those of the comets. Nonetheless, greater difficulties appear, when the eccentricity is rather small, especially when the variations of the anomaly $\Upsilon$ grow, however if the need should arise, this cure could be expected. These operations are chiefly hindered by the quantity $\frac{1}{w^{3}}$, unless it is possible to conveniently convert it into a series sufficiently convergent, whose whole integration would be despairing, and another option is not seen to be left over, unless from the single variations of these differential formulas versus very small intervals of time are defined, the task for the integration of these summations is compensated, and on another occasion I showed more details.

APPLICATION OF THIS THEORY TO THE MOTION OF THE MOON

$\mathrm{XXX}$ ) Let us consider that the center of the Earth is in $A$, which mass is $=A$, and considering the plane of the ecliptic in the figure, now, indeed, we have the Sun in $S$, which mass is $=B$, and for which, I place the line $A B$ directed to a fixed point in the sky such as to the First Star of Aries, being defined the following

longitude of the Sun or angle $B A N=\theta$

and distance of the Sun to the Earth or $A S=u$. 
These elements are defined for the Sun's motion: semi-major axis of the Sun's orbit $=a$, semi-latus rectum of the Sun's orbit $=b$, eccentricity of its orbit $=e$, and true anomaly $=v$, then $u=\frac{b}{1-e \cos v} \cdot{ }^{46}$ Then, in fact, considering the mean motion $^{47}$, if during an infinitesimal time the Sun traverses an angle $\mathrm{d} \tau$, then $u^{2} \mathrm{~d} \theta=a \mathrm{~d} \tau \sqrt{a b},{ }^{48}$ where $\mathrm{d} \theta=\mathrm{d} v$, and we wish to observe the motion only during the Sun's apogee. It is true that $b=a\left(1-e^{2}\right)$; then we have that

$$
u=\frac{a\left(1-e^{2}\right)}{1-e \cos v}, \text { and } \mathrm{d} \tau=\frac{\mathrm{d} \theta\left(1-e^{2}\right)^{\frac{3}{2}}}{(1-e \cos v)^{2}} \text { and also, } \mathrm{d} v=\mathrm{d} \theta, \text { approximately, }
$$

where it should be noted that once the eccentricity of the Sun's orbit is neglected, then $u=a$ and $\mathrm{d} \theta=\mathrm{d} \tau$.

XXXI) Be further the Moon in $Z$, which mass $=C$, and be defined $\frac{B}{A+C}=n$, knowing that $\frac{A+C}{A+B}=m$, and since the elementary angle $\mathrm{d} \tau$ is chosen from the mean motion of the Sun, such that $\mathfrak{A}=A$ and $\mathfrak{C}=B$; whence giving $\frac{B}{A+B}=m n$ or $m=\frac{1}{n}$, since the mass of the Sun can be considered infinitely larger than the mass of the Earth. Now for the location of the Moon it is established that

$$
\text { longitude of the ascending node or angle } B A N=\psi
$$

inclination of the orbit of the Moon in relation to the ecliptic or angle $Y O Z=\omega$ and argument of latitude or angle $N A Z=\sigma$

then, we have that:

$$
\text { longitude of the Moon }=\psi+\underbrace{\arctan (\tan \sigma \cos \omega)}_{\text {this ss:?he angle } N A Y}
$$

and

$$
\text { northern latitude }=\underbrace{\arcsin (\sin \sigma \sin \omega)}_{\text {this is the angle ZAY }} \text {. }
$$

Then, if the distance of the Moon to the Earth $A Z=v$ and the distance of the Moon to the Sun $S Z=w$, and having been already defined the two angles $\mu$ and $v$, such that

$$
\begin{gathered}
\cos \mu=\cos \sigma \cos (\theta-\psi)+\sin \sigma \cos \omega \sin (\theta-\psi) \\
\sin v=\sin \sigma \cos (\theta-\psi)-\cos \sigma \cos \omega \sin (\theta-\psi) \\
\text { then, } w^{2}=v^{2}+u^{2}-2 v u \cos \mu \text { or } w=\sqrt{v^{2}+u^{2}-2 v u \cos \mu} .
\end{gathered}
$$

\footnotetext{
${ }^{46}$ The negative sign in the denominator indicates that the reference direction $\theta=0$, points towards the center of the ellipse, and positive if that direction points away from the center.

${ }^{47}$ In orbital mechanics, mean motion is the angular speed required for a body to complete one orbit, assuming constant speed in a circular orbit which completes in the same time as the variable speed, elliptical orbit of the actual body.

${ }^{48}$ This result comes from Kepler's 2nd law.
} 
XXXII) From these considerations, if we consider that currently $v=\frac{p}{1-q \cos \Upsilon}$ denotes half the parameter of the lunar orbit, $q$ is its eccentricity, and the angle $\Upsilon$ is its true anomaly; such that now $q$ should be considered negative; then, in fact, being $\mathrm{d} \varnothing$ the angle described by the Moon around the Earth in the same time, during which the Sun traverses the angle $\mathrm{d} \tau$ in its mean motion. Hence, for defining the motion of the Moon, the following equations should be considered:

1) $v^{2} \mathrm{~d} \varnothing=a \mathrm{~d} \tau \sqrt{m a p}=a \mathrm{~d} \tau \sqrt{\frac{a p}{n}}$

2) $\mathrm{d} p=-2 n v^{3} u \mathrm{~d} \varnothing \sin v\left(\frac{1}{w^{3}}-\frac{1}{u^{3}}\right)$

3)

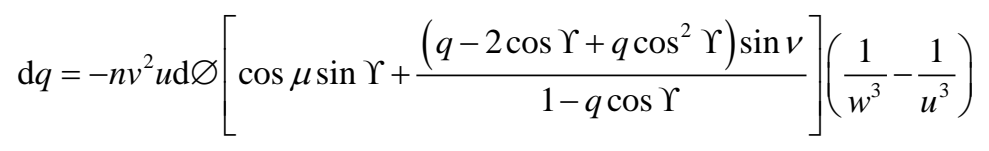

$$
+\frac{n v^{3} \mathrm{~d} \varnothing \sin \Upsilon}{w^{3}}
$$

4) $\frac{\mathrm{d} v}{v^{2}}=-\frac{q \mathrm{~d} \varnothing \sin \Upsilon}{p}$ or $\mathrm{d}\left(\frac{1}{v}\right)=+\frac{q \mathrm{~d} \varnothing \sin \Upsilon}{p}$

5)

$$
\begin{aligned}
\mathrm{d} \Upsilon= & \mathrm{d} \varnothing+\frac{n v^{3} \mathrm{~d} \varnothing \cos \Upsilon}{q w^{3}} \\
& -\frac{n v^{2} u \mathrm{~d} \varnothing}{q}\left[\cos \mu \cos \Upsilon+\frac{(2-q \cos \Upsilon) \sin v \sin \Upsilon}{1-q \cos \Upsilon}\right]\left(\frac{1}{w^{3}}-\frac{1}{u^{3}}\right)
\end{aligned}
$$

denoting $d \varnothing-d \Upsilon$ the instantaneous promotion of the line of apsides ${ }^{49}$ or the [promotion] of the Moon's apogee in its orbit:

6) $\mathrm{d} \psi=\frac{-n v^{3} u \mathrm{~d} \varnothing \sin \sigma \sin (\theta-\psi)}{p}\left(\frac{1}{w^{3}}-\frac{1}{u^{3}}\right)$

7) $\frac{\mathrm{d} \omega}{\sin \omega}=\frac{\mathrm{d} \psi}{\tan \sigma}=\frac{-n v^{3} u \mathrm{~d} \varnothing \cos \sigma \sin (\theta-\psi)}{p}\left(\frac{1}{w^{3}}-\frac{1}{u^{3}}\right)$

8) $\mathrm{d} \sigma=\mathrm{d} \varnothing-\mathrm{d} \psi \cos \omega=\mathrm{d} \varnothing+\frac{n v^{3} u \mathrm{~d} \varnothing \sin \sigma \cos \omega \sin (\theta-\psi)}{p}\left(\frac{1}{w^{3}}-\frac{1}{u^{3}}\right)$

XXXIII) Let us begin with the development of the expression $\frac{1}{w^{3}}-\frac{1}{u^{3}}$ : since it is obvious to be established that the distance $u$ is always much longer than the distance $v$, then we have that

$$
\frac{1}{w^{3}}=\left(u^{2}-2 v u \cos \mu+v^{2}\right)^{-1 / 2}=\frac{1}{u^{3}}+\frac{3 v \cos \mu}{u^{4}}+\frac{3 v^{2}\left(5 \cos ^{2} \mu-1\right)}{2 u^{5}} .
$$

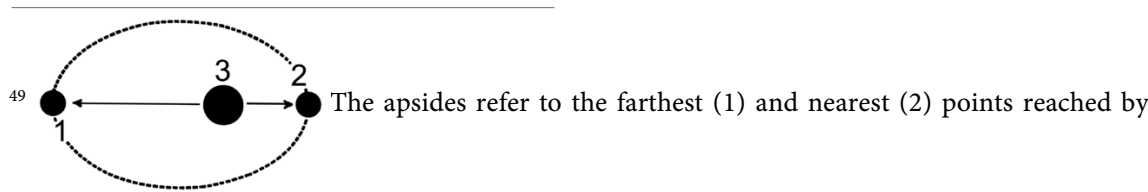

an orbiting planetary body ( 1 and 2 ) with respect to a primary, or host, body ( 3 ). The line of apsides is the line connecting positions 1 and 2. In the case of Moon and Earth, point 1, the farthest, is called apogee; and point 2, the nearest, is called perigee. 
And, therefore,

$$
\frac{1}{w^{3}}-\frac{1}{u^{3}}=\frac{3 v \cos \mu}{u^{4}}+\frac{3 v^{2}\left(5 \cos ^{2} \mu-1\right)}{2 u^{5}} .
$$

Then, the formulas n) 3 and n) 5 will transform into these expressions:

3)

$$
\begin{aligned}
\mathrm{d} q= & \frac{n v^{3} \mathrm{~d} \varnothing \sin \Upsilon}{u^{3}}\left[1-3 \cos ^{2} \mu+\frac{3 v \cos \mu}{2 u}\left(3-5 \cos ^{2} \mu\right)\right] \\
& -\frac{n v^{2} \mathrm{~d} \varnothing \sin v}{1-q \cos \Upsilon}\left(q-2 \cos \Upsilon+q \cos ^{2} \Upsilon\right)\left(\frac{3 v \cos \mu}{u^{3}}+\frac{3 v^{2}\left(5 \cos ^{2} \mu-1\right)}{2 u^{4}}\right) \\
\mathrm{d} \Upsilon= & \mathrm{d} \varnothing+\frac{n v^{3} \mathrm{~d} \varnothing \cos \Upsilon}{q u^{3}}\left[1-3 \cos ^{2} \mu+\frac{3 v \cos \mu}{2 u}\left(3-5 \cos ^{2} \mu\right)\right] \\
& -\frac{n v^{2} u d \varnothing \sin v \sin \Upsilon}{q(1-q \cos \Upsilon)}\left(\frac{3 v \cos \mu}{u^{3}}+\frac{3 v^{2}\left(5 \cos ^{2} \mu-1\right)}{2 u^{4}}\right)(2-q \cos \Upsilon)
\end{aligned}
$$

5)

Then, we have that:

$$
\mathrm{d} q \cos \Upsilon+q(\mathrm{~d} \varnothing-\mathrm{d} \Upsilon) \sin \Upsilon=2 n v^{2} n \mathrm{~d} \varnothing \sin v\left(\frac{3 v \cos \mu}{u^{3}}+\frac{3 v^{2}\left(5 \cos ^{2} \mu-1\right)}{2 u^{4}}\right)
$$

which is a quite simple expression that will be possible to use next.

XXXIV) Then, it should be noted furthermore that when the inclination $\omega$ is quite small, such that $\cos \omega=1-\frac{1}{2} \omega^{2}$, then, approximately, have that:

$$
\begin{gathered}
\cos \mu=\cos (\sigma-\theta+\psi)-\frac{1}{2} \omega^{2} \sin \sigma \sin (\theta-\psi) \\
\sin v=\sin (\sigma-\theta+\psi)+\frac{1}{2} \omega^{2} \cos \sigma \cos \omega \sin (\theta-\psi)
\end{gathered}
$$

\section{Conclusion}

"On the rectilinear motion of three bodies mutually attracting each other": this is considered the first work from which the three collinear Lagrange points could be obtained, where the parameter that controls the distances among the bodies was found to be given by a quintic function.

"Considerations on the motion of celestial bodies": one of the conclusions of this paper is that if the Moon were four times more distant from the Earth (either in conjunction or in opposition), a motion of this kind would have been possible to exist, such that the Moon would appear always connected to the Sun. In this paper, perhaps for the first time, Euler introduces an archaic form of a Fourier sine series expansion to describe the Moon's wagging motion. However, as Euler himself recognizes, the calculations turned out very tedious and led him to greatly simplify his model in order to obtain some numerical values for the phenomenon.

"An easy method for calculating the motion of celestial bodies perturbed in any manner avoiding astronomical computations": with few sketches to show 
the geometrical constructions envisaged by Euler-represented by several geometrical variables-it is hard to follow publication. The Translator, on trying to clear the way to the non-specialized reader, used the best of his abilities to add his own figures to the translation. In the latter part of the work, Euler particularizes his developments to the Moon, ending up with eight coupled differential equations for resolving the perturbed motion of this celestial body, which makes his claim of an "easy method" as being rather fallacious. Despite showing great analytical skills, Euler did not give indications on how this system of equations could be solved, which renders his efforts practically useless in the determination of the variations of the nodal line and inclination of the Moon's orbit.

\section{Conflicts of Interest}

The author declares no conflicts of interest regarding the publication of this paper.

\section{References}

Euler, L. (1766). Considerationes de motu corporum coelestium. Novi Commentarii Academiae Scientiarum Petropolitanae, 10, 544-558.

Euler, L. (1767). De motu rectilineo trium corporum se mutuo attrahentium. Novi Commentarii Academiae Scientiarum Petropolitanae, 11, 144-151.

Euler, L. (1768). Methodus facilis motus corporum coelestium utcunque perturbatos ad rationem calculi astronomici revocandi. Novi Commentarii Academiae Scientiarum Petropolitanae, 12, 129-165. 